\title{
An Evolutionary Approach to Financial Innovation
}

\author{
MARC OLIVER BETTZÜGE and THORSTEN HENS \\ University of Zurich
}

First version received June 1997; final version accepted March 2000 (Eds.)

\begin{abstract}
The purpose of this paper is to explain why some markets for financial products take off while others vanish as soon as they have emerged. To this end, we model an infinite sequence of CAPM-economies in which financial products can be used for insurance purposes. Agents' participation in these financial products, however, is restricted. Consecutive stage economies are linked by a mapping ("transition function") which determines the next period's participation structure from the preceding period's participation. The transition function generates a dynamic process of market participation which is driven by the percentage of informed traders and the rate at which a new asset is adopted. We then analyse the evolutionary stability of stationary equilibria. In accordance with the empirical literature on financial innovation, it is obtained that the success of a financial innovation, a mutation, depends on a sufficiently high trading volume, marketing, and new and differentiated hedging opportunities. In particular, a set of complete markets forming a stationary equilibrium is robust with respect to any further financial innovation while this is not necessarily true for a set of incomplete markets.
\end{abstract}

"There is no generally accepted theory of financial innovation, but some broad generalizations of the innovation process are possible. What matters is not the invention of a financial product or process (which is often obscure) but its diffusion through the market environment".

Ted Padolski (1987) in: The New Palgrave on Money and Finance: Financial Innovation and Money Supply (p. 68)

\section{INTRODUCTION}

The rapid development and unprecedented growth of international financial markets over the last two decades ${ }^{1}$ has become the object of intensive economic research. Literally every day an abundance of new financial assets is created on these markets; some of these new securities soon become standard instruments of financial trade, other ones disappear as quickly as they have emerged. This extensive process of financial innovation has led to deep structural changes in the scope and range of international financial markets.

This paper sets out from the claim that the development of financial markets must be regarded as a fundamentally dynamic process, connecting a series of basic historical changes. Therefore, we postulate that the analysis of the process of financial innovation requires treatment within a truly dynamic model. ${ }^{2}$ As the process of financial innovation is stimulated by a variety of different reasons, each leading to a different range of financial products, we think of causes for a specific innovation as a black box, assuming that, for whatever reason, new assets can perpetually enter the market. Then we consider an evolutionary selection process which distinguishes stable asset structures from such asset

1 For an assessment see Tufano (1989) and Miller (1992).

2. This need for a model describing the ongoing process of financial innovation is one of the main conclusions of the well known empirical research by Silber (1981) and Black (1986). 
structures which are likely to be modified by the innovation of some new financial product.

Building on the applied literature on this topic we assume that a high level of market participation generated by a certain financial security is one of the key determinants of its "survival" in the market. This observation has already been summarized by Black (1986), page 1:

"What do we mean by success or failure? A successful contract innovation attracts a lot of trading interest, draws many people to the pit and generates substantial order flow from off-the-floor participants. On the other hand, some contracts are introduced, and although a few people stand in the pit at the exchange, there is not enough interest or orders from other market participants to continue supporting the contract. The floor traders leave the pit and the exchange no longer provides floor space or price reports for the contract-it is a failure".

The empirical literature moreover suggests that the key determinants of market participation itself are trading volume and liquidity, ${ }^{3}$ degree of novelty and awareness. ${ }^{4}$

The essential ingredient of our evolutionary model is a standard (static) general equilibrium model with incomplete financial markets (GEI-model), where investors' market participation is assumed to be asymmetrically restricted. ${ }^{5}$ In such a model, investors are characterized not only by their respective endowments and their preferences (which we assume to be of the CAPM-type), but also by the subset of assets which they are able to trade on the market. We consider an intertemporal sequence of these stage economies, where we assume stationarity of all the standard GEI-characteristics. The proportion of investors trading in certain assets, however, varies over consecutive periods. This proportion can be interpreted as a "market participation rate". Using some transition function, which again is stationary, the evolution of market participation rates is modelled. Thus, starting from arbitrary initial conditions, an iterative dynamical process is defined allowing an analysis of its stationary equilibria (fixed points).

First, we give necessary and sufficient conditions for the existence of stationary equilibria, both for the cases of uniform participation and of mixed participation. In the former case, every asset is used by either everyone or by no one, while in the latter case there is some asset which is traded by some of the agents but not by all of them. We then analyse which of the stationary equilibria are robust with respect to small perturbations of the market participation rates. We investigate whether some new assets which have not been traded so far ("mutations") can succeed in being established in the market. We call stationary equilibria evolutionarily stable if there is an entry barrier for the participation rates of new assets, below which these assets will be pushed back out of the market. Moreover, a stationary equilibrium is called asymptotically stable, if for any sufficiently small perturbation of the corresponding market participation rates the dynamical process converges back to the stationary equilibrium. An asymptotically stable stationary equilibrium thus always is evolutionarily stable. In our model, the case of complete participation always is asymptotically stable if it happens to form a stationary equilibrium. Incomplete

3. This view is commonly held by applied as well as by theoretical economists (see Black (1986), Tufano (1989), Miller (1986), Duffie and Rahi (1995) or "The Economist" (1996)). According to Black (1986) traders prefer to "cross-hedge" the insurance possibilities offered by a new and hence relatively illiquid asset using well established liquid markets.

4. It is obvious that without being aware of the existence of a certain financial instrument one cannot trade in that instrument. According to Simon (1955), bounded rationality begins with the observation that individuals are not aware of all the choices available to them.

5. Such models were studied first by Merton (1987), Siconolfi $(1986,1989)$ and Balasko, Cass and Siconolfi (1990). Also see Polemarchakis and Siconolfi (1997). 
uniform participation phases are evolutionarily stable only with respect to such assets which do not generate a sufficiently high trading volume, which do not offer sufficiently new and differentiated hedging opportunities, or which are not supported by an adequate marketing effort. ${ }^{6}$ Moreover, mixed participation equilibria never are asymptotically stable in our model.

The issue of financial innovation has recently received a lot of attention in economic theory. ${ }^{7}$ The literature has mainly focused on optimal security design, i.e. on the innovator's decision problem, especially in the presence of asymmetric information. The models presented so far are of an inherently static nature. In general, only two time periods are considered. ${ }^{8}$ In the first period, imperfect competition between financial intermediaries (banks, brokering institutions, exchanges) is modelled, which determines some endogenous financial market structure. In a second step, this structure is used as the exogenous market structure for the well-known static GEI-financial market model. All of these models have to make rather strong assumptions on innovators' rationality. In particular, in these models financial intermediaries can perfectly anticipate every possible consequence of their alternative financial innovations. However, imperfect competition between financial intermediaries is a deep and very challenging problem, especially if one adds imperfect competition to a general equilibrium model. In fact, a recent paper by Corchón and Mas-Colell (1996) shows that games with imperfect competition can have indeterminate equilibria even if the corresponding GE-economy with perfect competition happens to have a unique equilibrium. We wish to avoid tackling this delicate issue. Instead, we take a complementary point of view by leaving the "obscure" process of asset creation to a black box and studying the diffusion of assets through the market environment. Yet, our approach can also serve as a dynamic alternative to the results obtained in the corresponding static models which depend on rather strong rationality assumptions, whereas our approach is based on principles of bounded rationality and does not need to consider intermediaries at all.

The paper is organized as follows. First, in Section 2, the static CAPM-economy with restricted participation is introduced and some useful properties of the stage economy are derived. Section 3 sets up the evolutionary process and defines corresponding intertemporal equilibrium and stability notions. Section 4 derives our main results illustrating them by some simple examples. Section 5 concludes the paper by giving an outlook on further research building on our evolutionary approach.

\section{THE STAGE ECONOMY}

\subsection{The RPGEI-model}

The first step of our evolutionary approach to financial innovation consists in the definition of a suitable stationary stage economy. The basis of the financial markets model

6. The role of marketing in CAPM-economies with restricted participation has previously been studied by Merton (1987). Similar to our interpretation, Merton considers the restriction in market participation as a lack of information about the existence of an asset. Merton (1987) then studies a monopolistic intermediary who chooses the optimal amount of marketing effort which balances the revenues from additionally informed agents and the cost of marketing.

7. For a summary see the survey article by Duffie and Rahi (1995) in the JET Symposium on Financial Innovation and the book by Allen and Gale (1994).

8. Models of the type discussed here are used for example in Cuny (1993), Duffie and Jackson (1989), Heller (1993), Allen and Gale (1994), Bisin (1998), Pesendorfer (1995) and Williamson (1996). 
at each stage of the evolutionary process is given by the standard GEI-model. For simplicity we assume that there is only one consumption good, which is divisible and perishable, and which is interpreted as a composite commodity. Moreover, there are two periods with uncertainty in the second period, which is modelled by $S$ possible states $s=1, \ldots, S$. Hence, consumption takes place on $S+1$ spot markets indexed by $s=0$ for the first period and by $s=1, \ldots, S$ for the second period. There are $I$ types of individuals $i=1, \ldots, I$, where each type consists of a continuum $[0,1]$ of identical agents. Hence, every agent is characterized by a utility-endowment combination $\left(U^{i}, \omega^{i}\right)$ for $i \in I$, where $U^{i}$ is a utility function mapping $\mathbb{R}^{S+1}$ into $\mathbb{R}$ and $\omega^{i} \in \mathbb{R}^{S+1}$.

Since the main point of this paper is not to investigate the most general allocation problem, the following rather strong assumption on the individuals' utility functions will be made.

Assumption (CAPM). $\quad U^{i}: \mathbb{R}^{S+1} \rightarrow \mathbb{R}$ is given by $U^{i}(x)=x_{0}+\sum_{s=1}^{s} \rho_{s}\left(x_{s}-\frac{1}{2} \gamma^{i}\left(x_{s}\right)^{2}\right)$, where $\gamma^{i}>0$ is the coefficient of type i's risk aversion.

For the analysis of GEI-economies with objective probabilities $\rho_{s}>0$ for $s=1, \ldots, S$, it is convenient to define the $\rho$-adjusted scalar product in $\mathbb{R}^{S}$ :

$$
\odot: \mathbb{R}^{s} \times \mathbb{R}^{s} \rightarrow \mathbb{R}, \quad \text { where } x \odot y:=\sum_{s=1}^{s} x_{s} \rho_{s} y_{s} .
$$

We will later make use of the norm $\|\cdot\|: \mathbb{R}^{s} \rightarrow \mathbb{R}$ defined by $\|y\|:=\sqrt{y \odot y}$, and we will call any two vectors $x, y \in \mathbb{R}^{s}$ "orthogonal" (with respect to $\rho$ ) if $x \odot y=0$. .

In the first period, agents can trade an exogenously given set of assets $j \in J$. Assets are "real", i.e. they pay off in units of the single consumption good. They can be distinguished by their payoff-vectors $A_{j} \in \mathbb{R}^{S}$. The set of all assets $J$ can then be viewed as an $S \times J$ matrix, i.e. $A=\left[A_{1}, \ldots, A_{J}\right] \in \mathbb{R}^{S \times J}$. The set of assets $J$ is the set out of which the evolutionary process draws its mutations. We think of $J$ to be a very large set allowing for all possible constellations of asset markets in which agents eventually participate. Since trading volume is one of the key driving forces of the evolutionary process, we will assume that all assets in $J$ have unit length according to the objective probability measure $\rho \in \mathbb{R}_{t+}^{S}$, i.e. $\left\|A_{j}\right\|=1$ for all $j \in J$. This normalization allows to give a meaningful definition of trading volume while not restricting the key characteristic of any GEI-model which is the market subspace $\langle A\rangle$, i.e. the column space of the asset matrix $A$. Markets will be said to be "complete" whenever the payoffs of the assets span the entire state space, i.e. whenever $\langle A\rangle=\mathbb{R}^{S}$. Otherwise, markets are incomplete. An asset with payoffs which are spanned by those of some other assets are called "redundant". Hence, an asset $A_{j}$ is redundant if there is a portfolio $\bar{\theta} \in \mathbb{R}^{J}$ such that $A_{j}=\sum_{k \neq j} \bar{\theta}_{k} A_{k}$.

In the standard GEI-model, every agent is able to trade each available asset. Here, agents can only use certain subsets of the assets. Considering the enormous amount of different assets available on today's financial markets this does not seem to be an implausible assumption. Especially for the discussion of the emergence of new assets (innovation), it seems rather reasonable to consider situations where- to start with only a small proportion of all the traders are both aware of this new trading opportunity and prepared to adopt it as a new trading instrument. ${ }^{10}$ Denoting by $\theta \in \mathbb{R}^{J}$ agent $i$ 's asset portfolio and by $q \in \mathbb{R}^{J}$ the vector of asset prices, ${ }^{11}$ we therefore consider the following decision problem

9. For these definitions and their role in CAPM-economies see for example Magill and Quinzii (1996, Chapter 3).

10. For a motivation of this assumption in the context of financial markets also see Merton (1987)

11. We use the common notation in which prices (quantities) are denoted by row (column) vectors. 
of an individual agent of type $i$ who is restricted to trade only such assets $j$ which are contained in a given subset $K \subset J$ of all the assets ${ }^{12}$

$$
\begin{array}{ll}
\left(P_{R P}^{i, K}\right) \max _{\substack{x \in \mathbb{R}^{s+1} \\
\theta \in \mathbb{R}^{j}}} U^{i}(x) \\
\text { s.t. } & \left(x-\omega^{i}\right)=\left(\begin{array}{c}
-q \\
A
\end{array}\right) \theta \\
& \theta_{j}=0 \quad \text { if } j \notin K .
\end{array}
$$

Observe that the definition of $\left(P_{R P}^{i, K}\right)$ implicitly assumes that the budget constraint $\left(x-\omega^{i}\right) \leqq\left(\begin{array}{c}-q \\ A\end{array}\right) \theta$ is satisfied with equality. This will be the case in equilibrium if either the market institutions do not allow for free disposal in the second period or if preferences are monotonic at the equilibrium consumption vector. Such a monotonicity condition can be ensured in the CAPM by choosing sufficiently small coefficients of individual risk aversion $\gamma^{i}>0$ for $i \in I^{13}$

Due to our assumptions, agents of the same type are distinguished only by the set $K$ describing the set of assets an individual is able to trade. Therefore, for every type of agents $i \in I$ and every set $K \subset J$ one can define the sets $J^{i, K}$ of all agents of type $i$ who can exactly trade in those assets contained in $K$. In the sequel, the economy will be specified such as to ensure that these sets are Borel-measurable for every $i \in I$ and $K \subseteq J$. Hence, one can define constants $p^{i}(K), i \in I, K \subseteq J$ by

$$
p^{i}(K):=\text { Lebesgue }- \text { measure }\left(J^{i, K}\right) .
$$

Thus, $p^{i}(\cdot)$ describes the distribution of the awareness restrictions imposed on agents of type $i$. Observe that each $p^{i}(\cdot)$ is a probability measure on $\mathscr{P}(J):=\{K \subseteq J\}$.

Lacking a clear understanding of how various endowment-utility combinations may affect market participation of an agent, we make two simplifying assumptions on the participation structures considered. First, we assume that all types of agents are identically restricted with respect to their market participation. This assumption will later allow us to obtain a simple solution for the equilibria of the stage economies.

Assumption (P). There is a $p$ such that $p=p^{i}$ for every $i \in I$.

Secondly, we restrict attention to such participation structures which are completely characterized by the proportion $r_{j}$ of agents knowing asset $j$. Since for a general participation structure $p$ the proportion of agents knowing asset $j$ is given by

$$
r_{j}=\sum_{K \subset J, j \in K} p(K),
$$

we therefore consider the participation structures $p \in[0,1]^{2 J}$ for which there exists a vector $r \in[0,1]^{J}$ such that

$$
p(K):=p^{r}(K)=\prod_{k \in K} r_{k} \prod_{\text {leK }}\left(1-r_{l}\right),
$$

for every $K \subseteq J$. We will denote such probability distributions by $p^{r}, r \in[0,1]^{J}$, in order to indicate the $J$-dimensional vector of factors corresponding to $p^{r}$, and we will frequently

12. For a decision-theoretic notion of awareness, which can be viewed as a foundation of this decision problem, see also Dekel, Lipman and Rustichini (1998).

13. It is well known, for example, that if markets are complete the assumption $1-\gamma^{i} \sum_{k=1}^{I} \omega_{s}^{k}>0$ for every $i \in I$ and every $s \in S$ suffices to show monotonicity of preferences on the relevant domain of allocations. Similar conditions can be found for the case of incomplete markets (see Siwik (2000)). 
refer to the vector $r$ as the "(market) participation structure" or "participation phase". ${ }^{14}$

Besides simplification of analysis, there is one important property of participation structures described by a phase $r \in[0,1]^{J}$ : In this case the participation rates of each of the individual assets are mutually uncorrelated in the sense that the knowledge of some particular asset does not favour the knowledge of any other asset. ${ }^{15}$ In practice it might often be the case that information about different assets is not acquired in such an independent way as postulated here; lacking a clear understanding, however, how the participation rate in some asset might affect the participation rate in another asset, this mutual independence seems the most natural assumption to start with.

We will often devote our attention to some relatively simple but important participation structures, namely those structures $p^{r}$ where $r_{j} \in\{0,1\}$ for all assets $j \in J$. With such a participation structure every agent is considering trading within the same non-empty subset of assets. Such structures are called uniform participation structures while the other structures are called mixed participation structures. Observe that uniform participation structures correspond to the standard GEI-situation without restricted participation.

With these definitions, the characteristics of the stage economies can be summarized as

$$
R P G E I=\left\{\mathbb{R}^{S+1}, A,\left(U^{i}, \omega^{i}\right)_{i \in I},\left(r_{j}\right)_{j \in J}\right\},
$$

where $R P G E I$ stands for a general equilibrium economy with incomplete markets and restricted participation.

\subsection{RPGEI-equilibria and associated trading volumes}

In an $R P G E I$-economy, the definition of RPGEI-equilibria can be stated as follows. Here, the superscript $(i, K)$ for $i \in I$ and $K \subseteq J$ is meant to indicate an arbitrary agent of type $i$ contained in the set $J^{i, K}$.

Definition 1. A tuple $(\stackrel{*}{x}, \stackrel{*}{\theta}, \stackrel{*}{q}) \in \mathbb{R}^{\left(I \times 2^{J}\right) \times(S+1)} \times \mathbb{R}^{\left(I \times 2^{J}\right) \times J} \times \mathbb{R}^{J}$ is called a restricted participation equilibrium with incomplete markets (RPGEI-equilibrium) at a given participation structure $r \in[0,1]^{J}$ if it satisfies the following conditions: ${ }^{16}$

(i) $\left.{ }^{*}{ }^{i, K},{ }^{*},{ }^{i, K}\right)$ solves $\left(P_{R P}^{i, K}\right)$ given $\stackrel{*}{q}$ for every $(i, K) \in I \times \mathscr{T}(J)$;

(ii) $\sum_{j \in I} \Sigma_{K \subseteq J} p^{r}(K) \cdot{ }^{*} \theta^{i, K}=0$, and

(iii) $\sum_{i \in I} \sum_{K \subseteq J} p^{r}(K) \cdot \stackrel{*}{x}^{i, K}=\sum_{i \in I} \omega^{i}$,

where $p^{r}(K)=\prod_{k \in K} r_{k} \prod_{l \notin K}\left(1-r_{l}\right)$ for every $K \subseteq J$.

The simplifying restrictions placed on the relevant participation structures as well as the assumption of quadratic utilities allow to explicitly solve for $R P G E I$-equilibria. In particular, this is a consequence of the following result which states that the innovation of a non-redundant asset in the quadratic CAPM does not change the equilibrium price

14. It is easy to see that for any $r \in[0,1]^{J}$ the vector $p^{r}$ defined by $p^{r}(K):=\Pi_{k \in K} \mathrm{r}_{k} \Pi_{l \notin K}\left(1-r_{l}\right)$, for every $K \subseteq J$, is in fact a probability measure on the power set $\mathscr{S}(J)$. By construction, the set of all such probability measures can be identified with $[0,1]^{J}$. In particular, it follows that this set is non-empty.

15. Consider the indicator variables $X_{j}: \mathscr{f}(J) \rightarrow\{0,1\}$ for $j \in J$ with $X_{j}(K)=1$ if and only if $j \in K$. It can easily be shown that $\operatorname{cov}\left[X_{j}, X_{k}\right]=0$ whenever $j \neq k$, provided $X_{j}$ and $X_{k}$ are distributed according to a probability measure of the form $p^{r}$.

16. Observe that the third condition in Definition 1 is redundant by the definition of the maximization problem $\left(P_{R P}^{i, K}\right)$. 
of the existing assets. ${ }^{17}$ As a further piece of notation we define $1:=(1, \ldots, 1) \in \mathbb{R}^{S}$, and, for any $S+1$-dimensional vector $x=\left(x_{0}, x_{1}, \ldots, x_{S}\right)^{T} \in \mathbb{R}^{S+1}$, we let $x_{1}:=\left(x_{1}, \ldots, x_{S}\right)^{T}$.

Lemma 1 (Market Partition Lemma). Given $\left\{A,\left(U^{i}, \omega^{i}\right)\right\}$, for every $K \subseteq J$ consider the standard GEI-economies parameterized by $r,\left\{\mathbb{R}^{s+1}, A,\left(U^{i}, \omega^{i}\right)_{i=1}^{I}, r\right\}$, where all the agents are restricted to trade exactly in those assets contained in $K$, i.e. where $r_{j}=1$ for $j \in$ $K$ and $r_{j}=0$ for $j \notin K$. It then follows that, the equilibrium asset prices do not vary with $K$, i.e. that the equilibrium asset prices ${ }_{q}^{*}(K)$ of these economies satisfy ${ }^{*}(K)={ }_{q}^{*}\left(K^{\prime}\right)$ for each $j \in K \cap K^{\prime}$.

Proof. Let $K \subseteq J$ be fixed, and consider a $G E I$-economy where all agents trade exactly in those assets contained in $K$. Without loss of generality ${ }^{18}$ assume that $K=J$. Then agent $i$ 's maximization problem can be rewritten as

$$
\max _{\theta^{i} \in \mathbb{R}^{J}} \omega_{0}^{i}-q \theta^{i}+\mathbb{1} \odot\left(\omega_{0}^{i}+A \theta^{i}\right)-\frac{\gamma^{i}}{2}\left(\omega_{1}^{i}+A \theta^{i}\right) \odot\left(\omega_{0}^{i}+A \theta^{i}\right) .
$$

Hence, the first order condition for this problem becomes

$$
\frac{1}{\gamma^{i}}(\mathbb{1} \odot A-q)=\left(\omega_{1}^{i}+A \theta^{j}\right) \odot A .
$$

Summing over types of agents, inserting the equilibrium condition $\sum_{i=1}^{I} \stackrel{*}{\theta}^{i}=0$, defining $\kappa:=\sum_{i=1}^{I}\left(1 / \gamma^{i}\right)$ and $\omega_{1}:=\sum_{i=1}^{I} \omega_{\mathrm{t}}^{i}$, and observing the fact that the unboundedness of the consumption sets guarantees an interior solution, then yields equilibrium prices $q^{*}$ as

$$
\stackrel{*}{q}=\left(1-\frac{1}{\kappa} \omega_{0}\right) \odot A .
$$

But then, obviously, $\stackrel{*}{q}_{j}$ does not depend on the pay-off structure of any asset other than asset $j$.

From the Market Partition Lemma one concludes that the price of an asset only depends on preferences and endowments but not on the other assets present in the economy. This property together with the $C A P M$-assumption suffices to prove the existence of a unique $R P G E I$-equilibrium ${ }^{19}$ as long as the assets in the economy are not redundant. In the presence of redundant assets, equilibrium allocations of consumption bundles still are unique but uniqueness of individual portfolio decisions obviously can no longer be expected.

Proposition 1. Suppose every agent can only trade in a non-redundant set of assets. Then there exists $a$ unique RPGEI-equilibrium.

Proof. Stratify the economy into those subeconomies consisting only of agents being able to trade exactly in the same non-empty subset of assets $K \subset J$. By Assumption (P) all those subeconomies have the same endowment-preference characteristics (which are just

17. This result can, for example, also be found in Oh (1994).

18. Note in particular that we do not assume that $J$ forms a complete set of assets.

19. While for more general preferences existence of an equilibrium can still be proven (see Siconolfi (1986, 1989) and Polemarchakis and Siconolf (1997)), uniqueness of such an equilibrium can no longer be expected. For a recent overview over uniqueness results for standard GEI-economies see Hens, Schmedders and Voss (1999). 
scaled differently). From the proof of the Market Partition Lemma, it is now clear that equilibria for these subeconomies exist, that they are unique, and, moreover, that asset prices are the same across all the subeconomies. Hence, the equilibrium prices $\stackrel{*}{q}$ of the subeconomies also form an equilibrium price vector for the global RPGEI-economy.

It remains to show that $q^{*}$ is the unique equilibrium price vector for the RPGEIeconomy. This, however, can be inferred from the monotonicity of the market demand function

$$
\theta(q):=\sum_{i=1}^{I} \sum_{K \subseteq J} p^{r}(K) \theta^{i, K}(q)
$$

which is defined on the set of no arbitrage-prices (see Magill and Quinzii (1966)). ${ }^{20}$ By assumption (CAPM), utility functions are quasi-linear in first period consumption. Quasilinearity of preferences implies monotonicity of individual asset demand functions $\theta^{i, K}$ (see the first-order condition derived in the proof of the Market Partition Lemma); and since aggregation preserves this property (see, for example, Hildenbrand and Kirman (1988)), market demand is, in fact, monotonic in prices in this economy. Finally, note that if $p^{r}(K)=0$ for some $K$ then the equilibrium consumption allocations are still unique but the equilibrium prices $\stackrel{*}{q}_{j}, j \in \kappa$ are arbitrary. In this case we choose

$$
\stackrel{*}{q}_{j}=\left(1-\frac{1}{\kappa} \omega_{1}\right) \odot A_{j}, \quad j \in \kappa .
$$

For a given participation structure $r$ and an associated $R P G E I$-equilibrium price vector $\stackrel{*}{q}(r)$, let now $\operatorname{vol}_{j}^{i}(K, r):=\left|\hat{\theta}_{j}^{i, K}(r)\right|$ be the $R P G E I$-equilibrium trading volume in asset $j$ as effected by an agent of type $i$ who is restricted to trade only in those assets contained in $K \subseteq K_{+}(r):=\left\{j \in J \mid r_{j}>0\right\} .^{21}$ For $\operatorname{vol}_{j}^{i}(K, r)$ to be well defined $K_{+}(r)$ is assumed to be a non-redundant set of assets. Aggregating over types of agents and all the possible restricting subsets of $J$, one then obtains total trading volume in asset $j$ as

$$
\begin{aligned}
v_{j}(r) & :=\sum_{i=1}^{I} \sum_{K \subseteq K_{+}(r)} p^{r}(K) \operatorname{vol}_{j}^{i}(K, r) \\
& =\sum_{K \subseteq K_{+}(p)} p^{r}(K) \operatorname{vol}_{j}(K, r),
\end{aligned}
$$

where $\operatorname{vol}_{j}(K, r):=\sum_{i=1}^{I} \operatorname{vol}_{j}^{i}(K, r)$. It should be noted that the summation can only be taken over sets contained in $K_{+}(r)$, since for other sets the associated asset demand is not defined at the participation structure $r$.

As a consequence of the Market Partition Lemma, the volume function has a very useful property: The aggregate trading volume of asset $j$ in the population of agents considering trade in the subset $K$ of assets, i.e. $\operatorname{vol}_{j}(K, r)$, is independent of the market participation phase $r$.

Proposition 2. For each $K \subseteq J$ and for every $j \in K$, there exists a positive constant $\alpha_{j}^{K}$ such that $\operatorname{vol}_{j}(K, r)=\alpha_{j}^{K}$ for any $r \in[0,1]^{J}$ with $K_{+}(r) \supseteq K$, where $K_{+}(r)$ is non-redundant.

Proof. Trading volume $\operatorname{vol}_{j}^{i}(K, r)$ of an individual trader knowing assets contained in $K$ only depends on the equilibrium prices $\stackrel{*}{q}(K)$. But prices $\stackrel{*}{q}(K)$ are independent of the participation structure by the previous Market Partition Lemma. Hence, independently of $r$ (i.e. independently of the relative sizes of the $2^{J}$ subeconomies restricted to trade

20. Here, $\theta^{i, K}(q)$ is the portfolio used in the solution to the maximization problem $\left(P_{R P}^{i, K}\right)$ for given prices $q$. $\stackrel{*}{q}(r)$.

21. Here, similar to the previous footnote, $\theta^{i, K}(r)$ is a solution to the maximization problem $\left(P_{R P}^{i, K}\right)$ for given 
assets in $K$ ), equilibrium prices for the $R P G E I$-economy are given by $\stackrel{*}{q} \equiv \stackrel{*}{q}(J)$. Therefore, $\alpha_{j}^{K}:=\operatorname{vol}_{j}(K, r)$ is constant over $r . \quad \|$

The simple participation structures considered in this paper then allow for an explicit characterization of the equilibrium trading volumes in our stage economies. In order to further simplify notation, we let $r_{\backslash j}:=\left(r_{1}, \ldots, r_{j-1}, r_{j+1}, \ldots, r_{J}\right) \in[0,1]^{J-1}$.

Corollary 1. For every asset $j \in J$ and every participation structure $r \in[0,1]^{J}$, trading volume is linear in $r_{j}, j \in K_{+}(r)$, i.e. there are constants $\mu_{j}$ such that $v_{j}(r)=\mu_{j} \cdot r_{j}$. Here, the constants $\mu_{j}$ are given by

$$
\mu_{j}:=\mu_{j}\left(r_{\backslash j}, \alpha_{j}\right):=\mu_{j}\left(r_{\backslash j},\left(\alpha_{j}^{K}\right)_{K \subseteq J}\right):=\sum_{K \subseteq J \backslash j\}}\left(\prod_{k \in K} r_{k}\right)\left(\prod_{l \notin K, l \neq j}\left(1-r_{l}\right)\right) \alpha_{j}^{K \cup\{j\}} .
$$

Proof. This follows from the fact that $p^{r}(K)=0$ if $j \notin K$, for some $j \in K_{+}(r)$. $\|$

Note that $\mu_{j}\left(r_{\backslash j}, \alpha_{j}\right)$ gives the trading volume of asset $j$ generated in the $R P G E I$ economy where every agent knows this asset (i.e. $\left.r_{j}=1\right)$ and participation in the other assets is described by $r_{y j}$. Observe also that the linearity of the volume function implies that volume is continuously differentiable on the entire set $[0,1]^{J}$ of participation phases.

Computing an asset's trading volume simplifies even further, when the asset structure is " $\rho$-orthogonal". 22

Lemma 2. Suppose $A=\left\{A_{1}, \ldots, A_{J}\right\}$ is such that $A_{j} \odot A_{k}=0$ for $j \neq k$ (" $\rho$-orthogonal asset structure"). Then $\alpha_{j}^{K}=\alpha_{j}^{I}$ if $j \in K$ and $\alpha_{j}^{K}=0$ otherwise.

Proof. Recall that $\kappa:=\sum_{i=1}^{I}\left(1 / \gamma_{i}\right)$ and that $\omega_{\mathbb{1}}$ denotes aggregate endowments in the second period. Let $A^{K}$ be the $S \times K$ submatrix of $A$ formed by the column vectors associated with the assets contained in $K$. Using the same line of reasoning as in the proof of the Market Partition Lemma, one can then derive equilibrium asset demand of a type $i$-agent trading only $K$ as

$$
\stackrel{*}{\theta}^{i}(K)=\frac{1}{\gamma^{i}}\left(\tilde{A}^{K}\right)^{-1}\left(A^{K}\right)^{T} \odot\left(\frac{1}{\kappa} \omega_{1}-\gamma^{i} \omega_{\mathrm{1}}^{i}\right),
$$

where $\tilde{A}^{K}=\left(A^{K}\right)^{T} \odot A^{k}$. Then $\rho$-orthogonality of the assets implies that $\tilde{A}^{K}$ is the $J$-dimensional identity matrix. It follows that

$$
\stackrel{*}{\theta}_{j}^{i}(K)=\frac{1}{\gamma^{i}} \frac{A_{j} \odot y^{i}}{\gamma^{i} A_{j} \odot A_{j}}=\frac{1}{\gamma^{i}}\left(A_{j} \odot y^{i}\right),
$$

where $y^{i}, i \in I$, is an $S$-dimensional vector independent of $K$.

From this, we can conclude that $\theta_{j}^{i}(K)=\theta_{j}^{i}\left(K^{\prime}\right)$ if $j \in K \cap K^{\prime}$, i.e. every agent being able to trade in asset $j$ will choose to trade the same amount of this asset independent of the other assets she is allowed to trade in. But then total trading volume in any asset has to be constant over the different markets associated with the subsets $K \subseteq J$.

In the case of $\rho$-orthogonal assets, we can therefore determine trading volume by a very simple formula.

Lemma 3. Suppose $A$ is a $\rho$-orthogonal asset structure. Then $v_{j}(r)=\alpha_{j}^{J} \cdot r_{j}$ for every asset $j \in J$ and every phase $r \in[0,1]^{J}$.

22. In Duffie and Jackson (1989), $\rho$-orthogonal asset structures emerge as the equilibrium outcome. 
Proof. Let $r \in[0,1]^{J}$. Then

$$
\mu_{j}\left(r_{\backslash j}, \alpha_{j}\right)=\alpha_{j}^{J} \sum_{K \subset \Omega j j\}}\left(\prod_{k \in K} r_{k}\right)\left(\prod_{l \notin K, l \neq j}\left(1-r_{l}\right)\right)=\alpha_{j}^{J},
$$

where the penultimate equality follows from Lemma 2 , and the ultimate one from simple multinomial algebra. II

Summarizing this discussion we conclude that in a $R P G E I$-economy with individuals characterized by standard $C A P M$-assumptions, and with a homogeneous distribution of trading restrictions (Assumption (P)), a unique equilibrium price exists since then prices are independent of the asset structure (by the Market Partition Lemma). Moreover, since asset demands in the incomplete market case are projections of the complete market demands (as can be seen from the proof of Lemma 2), asset demands also are independent of the asset structure if the assets are orthogonal.

The derivative of the trading volume with respect to the market participation is the key determinant for the stability of the evolutionary process we consider in the next section. Therefore, we provide the following two results which conclude the analysis of the stage economy.

Lemma 4. Let $r \in[0,1]^{J}$ and $K_{+}(r)$ be non-redundant. Then

$$
\partial_{r_{m}} v_{j}(r)=\left\{\begin{array}{cc}
\mu_{j}\left(r_{\backslash j}, \alpha_{j}\right) & \text { if } m=j \\
r_{j} \sum_{K \subset J \backslash j, m\}}\left(\prod_{k \in K} r_{k}\right)\left(\prod_{l \notin K, l \neq j, m}\left(1-r_{l}\right)\right)\left[\alpha_{j}^{K \cup\{j, m)}-\alpha_{j}^{K \cup\{j\}}\right] & \text { if } m \neq j
\end{array} .\right.
$$

Proof. For $m=j$, the result immediately follows from $v_{j}(r)=r_{j} \cdot \mu_{j}$ and from the fact that $\partial_{r_{j}} \mu_{j}=0$ by Corollary 1 . If $m \neq j$, then $\partial_{r_{m}} v_{j}=r_{j} \cdot \partial_{r_{m}} \mu_{j}$. Simple algebra now yields the formula stated in the lemma. II

Corollary 2. Let $r \in[0,1]^{J}$.

(i) If $A$ is $\rho$-orthogonal, then the Jacobian of $v$ is a diagonal matrix with diagonal entries $\partial_{r_{j}} v_{j}=\alpha_{j}^{J}$ for every $j \in J$.

(ii) If $r_{j}=0$ (i.e. if asset $j$ is not adopted within the economy), then the $j$-th row vector of the Jacobian of $v$ is given by the $j$-th unit vector multiplied by the positive scalar $\partial_{r_{j}} v_{j}=\mu_{j}\left(r_{{ }_{j}}, \alpha_{j}\right)$.

\section{Proof.}

(i) For a $\rho$-orthogonal asset structure we know that $\alpha_{j}^{K \cup\{j, m\}}-\alpha_{j}^{K \cup\{j\}}=0$ for any assets $j, m \in J$. Hence, our claim follows from Lemma 2 and Lemma 4 .

(ii) This is obvious from Lemma 4.

This finishes the description of the stage economy.

\section{THE EVOLUTIONARY PROCESS}

Having described the one-shot stage economy and having shown that equilibria for this stage economy do in fact exist and are unique, we now turn our attention to the evolutionary process generating a sequence of such stage economies, denoted by $R P G E I(t)$ for $t \in$ $\mathbb{N}_{0}$. In order to simplify matters as much as possible, all but one relevant variable are 
assumed to be stationary. In addition to the invariance of

- $R^{S+1}$ the commodity space,

- $U^{i}$ the utility functions,

- $\omega^{i}$ the endowments,

we also assume stationarity of

- $A$ the exogenous set of assets,

over the time path of the $R P G E I$-economies. Only individual market participation (i.e. the phases $\left(r_{1}(t), \ldots, r_{J}(t)\right)$ for $\left.t \in \mathbb{N}_{0}\right)$ will be updated in each period. Two issues now have to be addressed: how is a phase $r(t)$ translated into a phase $r(t+1)$, and which are the notions of stationarity and stability to be applied for the analysis of the resulting dynamical process. Both of these issues are discussed in the remainder of this section.

\subsection{The transition function}

First the relation between two consecutive time periods has to be established. For given utility functions and endowments the equilibrium itself only depends on the market participation. We define a stationary transition function

$$
g:[0,1]^{J} \rightarrow[0,1]^{J}, \quad r(t+1)=g(r(t))
$$

connecting consecutive time periods which translates the participation phase $r(t)$ into the participation phase $r(t+1)$. Hence, as can be inferred from the definition of $g$, transition is modelled in a Markovian way, i.e. market participation in period $t+1$ depends only on the market participation in period $t$ and remains unaffected by the history of the process in the periods preceding time $t^{23}$

We now assume that the transition function $g_{j}(r)$ of any asset $j$ consists of an awareness coefficient $d_{j}\left(r_{j}\right)$ and an adoption coefficient $a_{j}(r)$, i.e. we let

$$
r_{j}(t+1)=g_{j}(r(t))=d_{j}\left(r_{j}(t)\right) \cdot a_{j}(r(t)), \quad j \in J .
$$

This definition is motivated by the observation that in order to actively trade in an asset, agents must first become aware of that asset. Consequently, we introduce the notation $d_{j}\left(r_{j}(t)\right)$ for the proportion of agents knowing asset $j$ in period $t+1$. Whether traders being aware of an asset then also actually adopt it (by incorporating it into their decision problem) will be determined by the perceived attractiveness of the market as compared to already existing markets. In our model, the coefficient $a_{j}(r(t))$ thus denotes the percentage of traders being aware of asset $j$ and adopting it as a part of their maximization problem in period $t+1$. $^{24}$

To add more structure to the transition function we make explicit assumptions on the nature of the diffusion of awareness, $d_{j}$, and the percentage of actual adoption, $a_{j}$.

23. Note that this dynamic process is well-defined only if the $R P G E I$-equilibria are known to be unique (as is the case with the quadratic preferences considered here). With multiple equilibria in the stage economies, however, the transition process would no longer be well-defined as long as no explicit equilibrium selection mechanism were introduced.

24. The adoption of technical and social innovations has intensively been studied by psychologists and sociologists. There is overwhelming empirical evidence that adoption is not driven by a rational cost-benefit analysis but merely by rules of bounded rationality such as imitation (see Rogers (1995) and Rogers and Schoemaker (1971)). Following this observation, we do not base the adoption process on a rational cost-benefit analysis which might require anticipating the next period's equilibrium (which itself depends on who decides to participate in certain assets). 


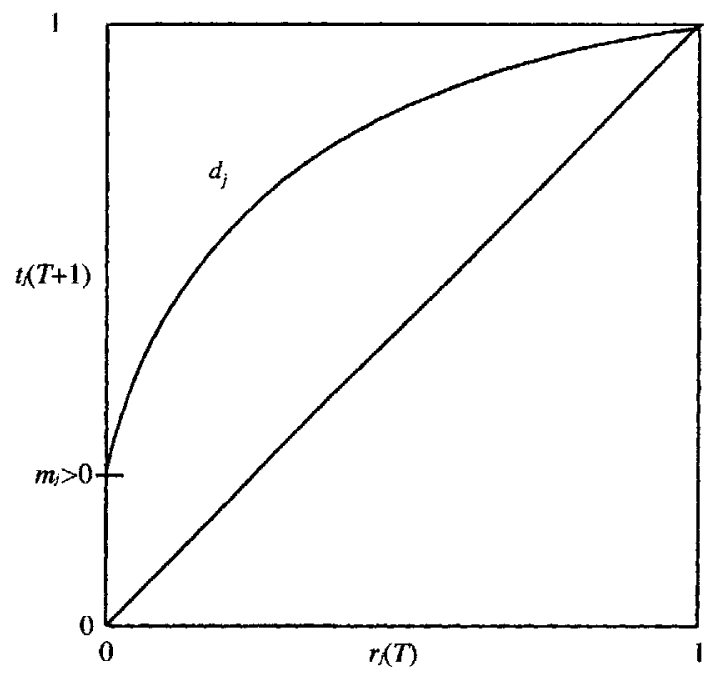

FIGURE 1

Illustration of $d_{j}$

First, we assume that $d_{j}\left(r_{j}\right)$ is a simple diffusion of information process, i.e.

$$
d_{j}\left(r_{j}\right)=r_{j}+m_{j}\left(1-r_{j}\right)+w_{j} r_{j}\left(1-r_{j}\right) \quad\left(0<m_{j} ; 0 \leqq w_{j} ; m_{j}+w_{j} \leqq 1\right) .
$$

According to this diffusion process, knowledge about the existence of asset $j$ can arise from three sources: $r_{j}$ is the percentage of people who remember asset $j$ from last period, ${ }^{25}$ $\left(1-r_{j}\right)$ is the percentage of previously uninformed agents who might get informed by public information ( $m_{j}>0$ is called the "marketing coefficient") or by private information ( $w_{j} \geqq 0$ is called the "word of mouth coefficient"). Awareness of an asset only depends on last period's awareness of this particular asset, but it is completely independent of the level of awareness of any of the other assets. Hence, there are no "spill-over"-effects with respect to the information about the existence of an asset.

The diffusion process $d_{j}\left(r_{j}\right)$ is a simple monotone and strictly concave mapping from $[0,1]$ into $[0,1]$ which has a unique stationary point $\bar{r}_{j}=1$. See Figure 1 for an illustration of $d_{j}{ }^{26}$

Whereas $d_{j}\left(r_{j}\right)$ is the percentage of agents being aware of asset $j, a_{j}$ is the "adoption coefficient" which determines the percentage of agents being aware of asset $j$ and actually considering to participate in the market for this asset in the next period $t+1$. Again we suggest a simple multiplicative structure: For $K_{+}(r)$ non-redundant let

$$
a_{j}(r)=\min \left\{1, f_{j}\left(v_{j}(r)\right) \cdot n_{j}(r)\right\},
$$

where $f_{j}$ represents a function measuring the relative "fitness" of an asset, whereas $n_{j}$ is supposed to reflect the degree of innovation an asset is offering compared to the other existing assets. As a consequence, this definition of the coefficients $a_{j}$ implies in general

25. Note that here we implicitly assume that agents do not forget.

26. Note that in the absence of the adoption coefficient $a_{j}$ the number of people being aware of the financial innovation will display an S-shaped time-path (upon iteration of our evolutionary process). This implication of the specification of the diffusion process $d_{j}$ is supported by the empirical literature on the diffusion of innovations (see Rogers (1995)). 
that the transition function for an asset $j \in J$ is a non-trivial function of the entire participation phase $r$ : whereas $d_{j}$ only depends on $r_{j}$, the adoption coefficients $a_{j}$, and hence the transition functions $g_{j}$, depend on $r_{1}, \ldots, r_{J}$. Thus the transition dynamics is inherently multi-dimensional, and there are potential "spill-over"-effects between the assets.

The concept of fitness of an asset should reflect the expected gains from participating in this asset, and we take the trading volume to be an indicator for those gains. ${ }^{27}$ To fix ideas we will simply assume that $f_{j}\left(v_{j}\right)=v_{j}$. It should be noted that the assumed linearity of $f$ will have a considerable effect on the results obtained in the following section, since it precludes complicated functional forms of the composite transition function $g$.

There are at least three arguments made in the literature which support the assumption that the fitness of a financial asset is positively correlated with the volume it generates. ${ }^{28}$ Firstly, trading volume is often interpreted as a proxy for the liquidity of a financial asset (for a theoretical foundation of this argument see for example Hopenhayn and Werner (1996) or Pagano (1989a)). Liquid assets are, ceteris paribus, considered to be more attractive to investors than illiquid ones for three reasons: They can better be realized at short notice (Grossmann and Miller (1988)), they are subject to a reduced influence of strategic behavior-which can either be due to asymmetric information as in Kyle $(1985,1989)$ or Admati and Pfleiderer (1988), or due to imperfect competition as in Pagano (1989a) - and they are less volatile (see e.g. Pagano (1989b)). Secondly, a number of studies have stressed that trading volume is a major factor governing the actions of financial intermediaries. Such intermediaries, for example exchanges or brokers, are often (directly or indirectly) compensated by some form of transaction fees which are proportional to the trading volume; hence, these intermediaries attempt to find and to promote such financial assets which generate high trading volumes. For models in support of this argument see for example Duffie and Jackson (1989), Cuny (1993), Hara (1995), or Bisin (1999), ${ }^{29}$ Finally, a third argument can be made in favour of interpreting an asset's trading volume as its fitness: As Bikhchandani, Hirshleifer and Welch (1998) have pointed out, herding behaviour is an important phenomenon in the presence of bounded knowledge and asymmetric information. In this sense, high trading volume can here be interpreted as a signal that an asset is widely and successfully used, and which therefore attracts new consumers who had so far not adopted this asset.

Finishing the specification of the transition function, the factor $n_{j}(r)$ is supposed to measure the degree of innovation in the payoffs of asset $j$ relative to the other assets already established where $n_{j}(r)=1$ if $K_{+}(r)=\{j\}$ or $K_{+}(r)=\varnothing$. To be more precise, let a participation rate $\bar{r}$ be given and assume that $K_{+}(\vec{r})$ forms a non-redundant set of assets. Then one can decompose the payoff $A_{j}$ for any asset $j \in J$ into a spanned component $S_{j}$ and an innovation component $I_{j}$, i.e. $A_{j} \equiv I_{j}+S_{j}$, where $S_{j}$ is the $\rho$-orthogonal projection of $A_{j}$ onto the span of the otherassets already traded at $\bar{r}$. Say $S_{j}=\sum_{k \in K_{+}(r) \backslash\{j\}} A_{k} \bar{\theta}_{k}$, where $\bar{\theta}$ is defined as the unique solution to the optimization problem.

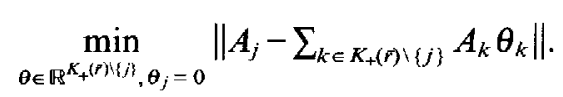

27. Obviously, if the preceding trading volume was zero, then no trader was able to gain anything on that market. Moreover, the higher the trading volume the more gains, in general. Hence, the fitness function should be starting at 0 and being monotonically increasing.

28. Black (1986) even defines the success of a financial innovation by the trading volume attracted.

29. Observe that-in order to keep things separate-we have not incorporated this effect into the "marketing coefficient" $m_{j}$ (which we assumed to be exogenous and fixed), but that we have rather amalgamated all volumerelated effects into the adoption coefficient $a_{j}$. 
In the sequel we will assume that this "innovation" coefficient has the following simple shape

$$
n_{j}(r)=\sqrt{\left\|I_{j}\right\|^{2}+\beta_{j}(r)\left\|S_{j}\right\|^{2}},
$$

where $\beta_{j}(r)=r_{j} /\left(r_{j}+\sum_{\left.k \in K_{+}(\tilde{r}) \backslash j\right\}} r_{k}\left|\bar{\theta}_{k}\right|\right)$ if the denominator is different from zero, and $\beta_{j}(r)=1$ otherwise

For a motivation of this specific assumption on $n_{j}$ observe that in a model with restricted participation the degree of "innovativeness" of an asset depends on two factors: On the one hand, and as acknowledged by the literature on financial innovation, ${ }^{30}$ it depends on the level to which asset $j$ contributes to the spanning opportunities available in the economy. On the other hand, the degree of novelty of an asset depends on the relative participation in asset $j$ compared to the participation in the other assets. This has already been pointed out by Black (1986) who stresses that the availability of well-established "cross-hedging" opportunities for a new asset is one of the major reasons for the failure of this asset. Our formulation of the function $n_{j}$ therefore measures the "spanning factor" by $\left\|I_{j}\right\|$, and captures the "cross-hedging" factor by introducing the coefficient $\beta$.

Regarding the properties of the function $n_{j}$, note that $\beta_{j} \in[0,1]$ and hence $n_{j} \in[0,1]$ by our normalization assumption $\left\|A_{j}\right\|=1$ for all $j \in J$. Moreover, $n_{j}$ is non-decreasing in $r_{j}$ and continuously differentiable in $r{ }^{31}$ Furthermore, for a $\rho$-orthogonal asset $A_{j}$, it is obtained that $n_{j}=1$ for all $r_{j} \in[0,1]$. Finally, if $A_{j}$ has zero participation and is redundant to some assets with positive market participation, then $n_{j}=0$. These properties of $n_{j}$ are, in fact, the only properties which will be used to derive our general results. The examples given in Subsection 4.2 are computed using the specific function $n_{j}$ defined above.

\subsection{Notions of stationarity and stability}

We now first have to look for steady states of the evolutionary process. These are captured by the following notion of a fixed point of the transition mapping defined by $g$. Our notion of stationarity thus describes a situation of market participation rates (and trading volumes) that sustain themselves over time, allowing for both high participation in some assets and low participation in other assets.

Definition 2. A participation phase $\bar{r} \in[0,1]^{J}$ is a stationary equilibrium of the evolutionary process if $\bar{r}=g(\vec{r})$.

From the definition of the transition function $g$ it is obvious that $r=(0, \ldots, 0)$ always is a stationary equilibrium.

We continue by investigating the stability of stationary equilibria by analysing which set of assets is robust with respect to some ongoing process of financial innovations. First, we define stability of a set of assets with respect to the further introduction of new assets, i.e. financial innovations.

Definition 3. A stationary equilibrium $\tilde{r} \in[0,1]^{J}$ with $\bar{r}_{j}=0$ is evolutionarily stable with respect to the innovation of asset $j$ if the following holds: There exists some $\varepsilon_{j} \in(0,1)$ such that for every $\tilde{r}_{j} \in\left(0, \varepsilon_{j}\right)$ the sequence $r(t+1)=g(r(t))$, with $r(0)=\left(\bar{r}_{\backslash j}, \tilde{r}_{j}\right), t=$ 
$0,1, \ldots$ converges to $\bar{r}$. Moreover, a stationary participation phase is evolutionarily stable if it is evolutionarily stable with respect to any innovation.

Thus, a stationary participation structure is evolutionarily stable if the traded assets can be protected from "mutants" by certain entry barriers for the initial proportion of traders participating in the new asset markets. Note that if the uniform participation phase $\bar{r}=(1, \ldots, 1)$ is a stationary equilibrium, it is also evolutionarily stable since no more entrants can appear.

Similarly, we ask whether some asset market "remains perfect", i.e. whether an asset market with complete participation has a tendency to return to the situation of complete participation if its participation rate is reduced slightly below 1 .

Definition 4. A stationary participation phase $\bar{r} \in[0,1]^{J}$ with $\bar{r}_{j}=1$ remains perfect with respect to asset $j$ if the following holds: There exists some $\varepsilon_{j} \in(0,1)$ such that for every $\vec{f}_{j} \in$ $\left(1-\varepsilon_{j}, 1\right)$ the sequence $r(t+1)=g(r(t))$, with $r(0)=\left(\bar{r}_{\backslash j}, \tilde{r}_{j}\right), t=0,1, \ldots$ converges to $\bar{r}$. Moreover, a stationary participation phase remains perfect if it remains perfect with respect to any asset.

A stronger stability requirement than evolutionary stability and remaining perfect is given by

Definition 5. A stationary participation phase $\bar{r} \in[0,1]^{J}$ is asymptotically stable with respect to the subsets of assets $K \subset J$ if there exists some $\varepsilon>0$ such that for every $\tilde{r} \in[0,1]^{J}$ with $\tilde{r}_{j}=\bar{r}_{j}, j \notin k$ and with $\|\tilde{r}-\bar{r}\|<\varepsilon$ the sequence, $r(t+1)=g(r(t))$ with $r(0)=\tilde{r}, t=0,1, \ldots$ converges to $\bar{r}$. Moreover, a stationary phase is asymptotically stable if it is asymptotically stable with respect to $k=J$.

Note that participation structures which are asymptotically stable also are evolutionarily stable and remain perfect for every asset $j$ while the opposite implication in general fails to hold. The concepts differ, since evolutionary stability and remaining perfect only require robustness with respect to changes in the participation rates of single assets while asymptotic stability considers small deviations from the market participation rate in any possible direction.

\section{MAIN RESULTS}

\subsection{Analytical results}

We first introduce specific one-dimensional functions $h_{j}$ for every $j \in J$, which serve to greatly simplify much of the following exposition. In fact, for every $j \in J$ and for every (fixed) $r_{\backslash,} \in[0,1]^{J-1}$ we define

$$
\begin{aligned}
h_{j}\left(r_{j}\right) & :=g_{j}\left(r_{\backslash j}, r_{j}\right) \\
& =\left\{\begin{array}{ll}
d_{j}\left(r_{j}\right) \cdot n_{j}(r) \cdot \mu_{j}\left(r_{\backslash j}, \alpha_{j}\right) \cdot r_{j} & \text { if } \mu_{j}\left(r_{\backslash j}, \alpha_{j}\right) n_{j}(r) r_{j}<1 . \\
d_{j}\left(r_{j}\right) & \text { otherwise }
\end{array} .\right.
\end{aligned}
$$

Thus $h_{j}$ is the one-dimensional function governing the dynamics of $r_{j}$ if the participation rates, $r_{\backslash j}$, of the other assets were to be kept fixed. It will turn out in the sequel that in spite of the potential spill-over effects present in the economy (due to the construction of $n_{j}$ and $\mu_{j}$ which may depend on the entire vector $r$ ), all the results for the existence 

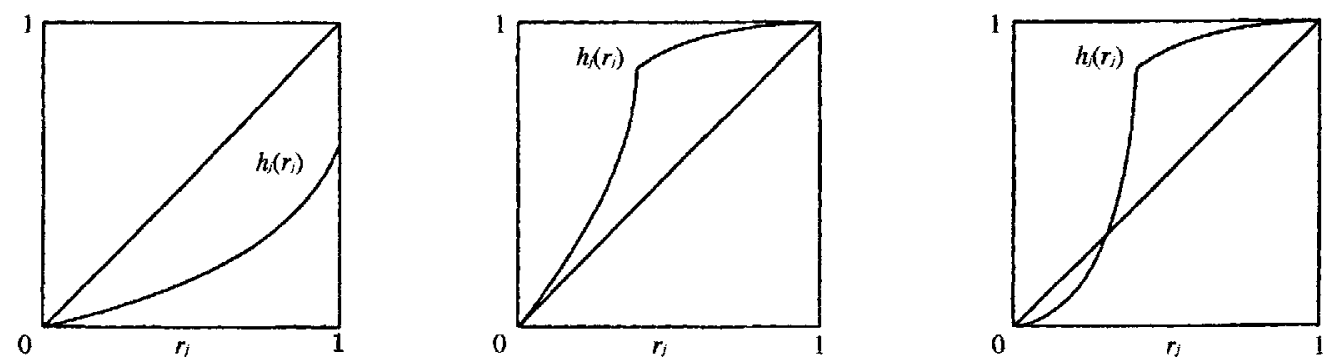

Figure 2

Generic shapes of the function $h_{j}$ (Lemma 5$)$

and stability of stationary points derived below can be deduced from the shape of $h_{j}$. The following lemma shows that-except for irregular cases $-h_{j}$ essentially takes three possible shapes, which are depicted in Figure 2.

Lemma 5. Let $j \in J$ and $r_{\backslash j} \in[0,1]^{J-1}$ be given, and let $\mu_{j}=\mu_{j}\left(r_{\backslash_{j}}, \alpha_{j}\right)$. Then $h_{j}\left(r_{j}\right):=g_{j}\left(r_{\vee j}, r_{j}\right)$ has the following properties:

(i) $h_{j}(0)=0$. Furthermore, $h_{j}(1)=1$ only if $n_{j}\left(r_{\backslash j}, 1\right) \mu_{j} \geqq 1$.

(ii) If $n_{j}\left(r_{\backslash j}, 1\right) \mu_{j}<1$ then $h_{j}\left(r_{j}\right)<r_{j}$ for every $r_{j} \in(0,1]$ and $h_{j}^{\prime}(0)<1$.

(iii) If $n_{j}\left(r_{\backslash j}, 1\right) \mu_{j}>1$ and $m_{j} n_{j}\left(r_{\backslash j}, 0\right) \mu_{j}>1$ then $h_{j}(1)=1, h_{j}^{\prime}(0)>1$, and $h_{j}\left(r_{j}\right)>r_{j}$ for all $r_{j} \in(0,1)$.

(iv) If $n_{j}\left(r_{\backslash j}, 1\right) \mu_{j}>1$ and $m_{j} n_{j}\left(r_{\backslash j}, 0\right) \mu_{j}<1$ then $h_{j}(1)=1, h_{j}^{\prime}(0)<1$, and there exists $a$ unique $\bar{r}_{j} \in(0,1)$ such that $h_{j}\left(\bar{r}_{j}\right)=\tilde{r}_{j}$. Moreover, in this case, $h_{j}$ is differentiable at $\bar{r}_{j}$ and $h_{j}^{\prime}\left(\bar{r}_{j}\right)>1$.

Proof.

(i) This follows directly from inserting $r_{j}=0$ and $r_{j}=1$ into the definition of $h_{j}$.

(ii) In this case

$$
h_{j}(1)=d_{j}(1) n_{j}\left(r_{\backslash j}, 1\right) \mu_{j}<1,
$$

hence $h_{j}$ ends below the diagonal. Since therefore

$$
h_{j}\left(r_{j}\right) \leqq d_{j}\left(r_{j}\right) n_{j}\left(r_{\backslash j}, r_{j}\right) \mu_{j} \cdot r_{j} \leqq d_{j}(1) n_{j}\left(r_{\backslash j}, 1\right) \mu_{j} \cdot r_{j}<r_{j},
$$

it follows that $h_{j}$ never crosses the diagonal in the open interval $(0,1)$. Moreover, one easily computes the derivative at $r_{j}=0$ as

$$
h_{j}^{\prime}(0)=d_{j}(0) n_{j}\left(r_{\vee j}, 0\right) \mu_{j}<d_{j}(0)=m_{j} \leqq 1 .
$$

(iii) Here, $h_{j}(1)=d_{j}(1)=1$ by construction. Now consider the function $\tilde{h}_{j}$ defined as

$$
\tilde{h}_{j}\left(r_{j}\right):=d_{j}\left(r_{j}\right) n_{j}\left(r_{\backslash j}, r_{j}\right) \mu_{j}\left(r_{\backslash j}, \alpha_{j}\right)
$$

for every $r_{j} \in[0,1]$. Clearly, $\tilde{h}_{j}$ is strictly increasing, since $d_{j}$ is strictly increasing and $n_{j}$ is non-decreasing. Under the assumptions stated it follows that $\tilde{h}_{j}(0)>1$. Hence, $\bar{h}_{j}\left(r_{j}\right)>1$ for every $r_{j} \in[0,1]$. Noting that $h_{j}\left(r_{j}\right)=\min \left\{h_{j}\left(r_{j}\right) \cdot r_{j}, d_{j}\left(r_{j}\right)\right\}>r_{j}$ for every $r_{j} \in(0,1)$, then proves that $h_{j}$ cannot cross the diagonal in the open interval $(0,1)$. Furthermore, the assumptions imply that $h_{j}^{\prime}(0)=$ $m_{j} n_{j}\left(r_{\backslash j}, 0\right) \mu_{j}>1$. 
(iv) Again, $h_{j}(1)=d_{j}(1)=1$ follows by construction. Now, for the function $\tilde{h}_{j}$ defined above, one obtains that $\tilde{h}_{j}(0)<1$ and $\tilde{h}_{j}(1)>1$. Hence, due to the strict monotonicity of $\tilde{h}_{j}$, there must be a unique point $\bar{r}_{j} \in(0,1)$ such that $\tilde{h}_{j}\left(\bar{r}_{j}\right)=1$. But in this case $h_{j}\left(\bar{r}_{j}\right)=\tilde{h}_{j}\left(\bar{r}_{j}\right) \cdot \bar{r}_{j}=\bar{r}_{j}$, and $\bar{r}_{j}$ is unique in $(0,1)$ as claimed.

In the case considered, the derivative of $h_{j}$ at $r_{j}=1$ satisfies $h_{j}^{\prime}(1)<1$. But then, at $\bar{r}_{j}, h_{j}$ must cross the diagonal from below. Since $d_{j}\left(\bar{r}_{j}\right)>\bar{r}_{j}$ implies $a_{j}\left(\bar{r}_{j}\right)<1$, it follows that $h_{j}$ is differentiable at $\bar{r}_{j}$. Hence, $h_{j}^{\prime}\left(\bar{r}_{j}\right)>1$.

To see what is driving this result consider the role of $a_{j}$ : Either it never becomes constant, then $h_{i}$ is stationary only in $r_{j}=0$. If, however, it does become constant then, in a neighbourhood of $r_{j}=1, h_{j}$ is identical with $d_{j}$ which has a simple shape and which strictly lies aboves the diagonal.

Therefore, Lemma 5 completely characterizes the possible shapes of $h_{j}$ in all the cases where $m_{j} n_{j}\left(r_{\backslash j}, 0\right) \mu_{j} \neq 1$ and $n_{j}\left(r_{\backslash j}, 1\right) \mu_{j} \neq 1$ ("regularity conditions"). Since we are only concerned with phases $r_{\backslash j}$ which are part of a stationary equilibrium, it suffices to show that these regularity conditions are met for all stationary points. As the next proposition demonstrates, this is in fact true for almost all endowments $\omega=\left(\omega^{1}, \ldots, \omega^{I}\right) \in \mathbb{R}^{(S+1) I}$.

Lemma 6. For all endowment distributions, except for some set $C \subset \mathbb{R}^{(S+1) I}$ of Lebesque-measure zero, the regularity conditions $m_{j} n_{j}\left(\bar{r}_{\backslash j}, 0\right) \mu_{j} \neq 1$ and $n_{j}\left(\bar{r}_{\backslash j}, 1\right) \mu_{j} \neq 1$, where $\mu_{j}=\mu_{j}\left(\bar{r}_{\backslash j}, \alpha_{j}\right)$, hold at all stationary equilibria $\bar{r} \in[0,1]^{J}$ with $K_{+}(\bar{r})$ forming a non-redundant set of assets.

Proof..$^{32}$ Let $\bar{r} \in[0,1]^{J}$ be a stationary equilibrium. As in the Market Partition Lemma, consider the GEI-economies $\operatorname{GEI}(\bar{r}, K)=\left\{\mathbb{R}^{(S+1)}, A(\bar{r}, K),\left(U^{i}, \omega^{i}\right)_{i=1}^{I}\right\}$, where $A(\bar{r}, K)$ is a submatrix of $A$, consisting only of assets $j \in K \subseteq K_{+}(\bar{r})$ with positive participation rates. Equilibrium trading volumes are

$$
\stackrel{*}{*}^{i}(\omega)=\frac{1}{\gamma^{i}}\left(A(\bar{r}, K)^{T} \odot A(\bar{r}, K)\right)^{-1} A(\bar{r}, K)^{T} \odot\left(\frac{1}{\kappa} \omega_{1}-\gamma^{i} \omega_{1}^{i}\right), \quad i=1, \ldots, I,
$$

where $\kappa=\sum_{i=1}^{I}\left(1 / \gamma^{i}\right)$ and $\omega_{1}=\sum_{i=\theta_{i}}^{I} \omega_{\mathbb{1}}^{i}$. Hence, $\hat{\theta}^{*}(\omega)$ is a continuously differentiable function for $\omega \in \mathbb{R}^{(S+1) I}$ and rank $\partial_{\omega} \theta^{i}(\omega)=|K|$, i.e. $\theta^{i}(\omega)$ is "controllable" by $\omega$. Hence, by the parametric transversality theorem, for all $\omega$ except for a set $C$ of measure zero, $\vec{\theta}_{j}^{i}(\omega) \neq 0$ so that the aggregate trading volume $\alpha_{j}^{K}(\omega)=\sum_{i=1}^{I}\left|\hat{\theta}_{j}^{*}(\omega)\right|$ is continuously differentiable and controllable by $\omega$, i.e. rank $\partial_{\omega}\left(\alpha_{1}^{K}(\omega), \ldots, \alpha_{J}^{k}(\omega)\right)=|K|$.

Therefore, for all $\omega$, except for a set of measure zero, $n_{j}(\bar{r}) \mu_{j}\left(\bar{r}_{\backslash j}, \alpha_{j}^{K}\right) \neq 1$ for all $j \in J$ and for all $K \subseteq K_{+}(\bar{r})$. A similar argument can be applied for the second regularity condition $m_{j} n_{j}\left(r_{\backslash j}, 0\right) \mu_{j}\left(\bar{r}_{\backslash j}, \alpha_{j}^{K}\right) \neq 1$, noting that $n_{j}$ is continuously differentiable in $r_{\backslash j}$. \|

The stability properties of the evolutionary process are determined by the eigenvalues of the Jacobian of the transition function $g:[0,1]^{J} \rightarrow[0,1]^{J}$ evaluated at the stationary equilibria. Therefore, we need to clarify whether $g$ is differentiable at every stationary phase $\bar{r} \in[0,1]^{J}$, where, as has been noted before, for stationary phases in the boundary of $[0,1]^{J}$ the derivative has to be taken only in any direction pointing to the interior of $[0,1]^{J}$. Note that the transition function $g$ need not be differentiable at a point where $n_{j} v_{j}=1$.

32. This proof uses some parametric transversality theorems (for a precise statement and a thorough discussion thereof see Magill and Quinzii (1996)). 
Proposition 3. For all endowment distributions, except for some set $C$ of measure zero, the transition function $g:[0,1]^{J} \rightarrow[0,1]^{J}$ is continuously differentiable at all stationary equilibrium phases $\bar{r} \in[0,1]^{J}$ with $K_{+}(\bar{r})$ forming a non-redundant set of assets.

Proof. By construction, $d_{j}\left(r_{j}\right)$ and $n_{j}(r)$ are continuously differentiable. Corollary 1 has demonstrated that the volume function $v_{j}(r)$ is continuously differentiable as well. Hence, it remains to argue that, generically in endowments, $n_{j}(\bar{r}) v_{j}(\bar{r})=$ $n_{j}(\bar{r}) \mu_{j}\left(\bar{r}_{\backslash j}, \alpha_{j}\right) \bar{r}_{j} \neq 1$ for all stationary equilibria $\bar{r}$ under consideration. Now $g_{j}(\bar{r})=\bar{r}_{j}$ and $n_{j}(\vec{r}) v_{j}(\bar{r})=1$ imply $d_{j}\left(\bar{r}_{j}\right)=\bar{r}_{j}$ and, hence, $\vec{r}_{j}=1$. Therefore, the claim follows as a corollary from the previous lemma. \|

After these preliminaries we can characterize stationary equilibria $\bar{r}$ in terms of properties of their coordinates $\bar{r}_{j}$ for $j \in J$.

Theorem 1. Suppose $K_{+}\left(\bar{r}_{\backslash j}, 1\right)$ forms a non-redundant set of assets, and let $\vec{r}$ be $a$ stationary equilibrium. Let $\mu_{j}=\mu_{j}(\bar{r})$. Then

(i) $\bar{r}_{j}=1$ only if $n_{j}(\bar{r}) \mu_{j} \geqq 1$.

(ii) Moreover, at $\bar{r}$, let the economy satisfy the regularity conditions, i.e. let $m_{j} n_{j}\left(\bar{r}_{\vee j}, 0\right) \mu_{j} \neq 1$ and $n_{j}\left(\bar{r}_{\backslash j}, 1\right) \mu_{j} \neq 1$. Then $\bar{r}_{j} \in(0,1)$ only if

$$
n_{j}\left(\bar{r}_{\backslash j}, 0\right) \mu_{j} m_{j}<1<n_{j}\left(\bar{r}_{\backslash j}, 1\right) \mu_{j} .
$$

Such a mixed participation phase is unique in $(0,1)$.

Proof. A stationary equilibrium $\bar{r} \in[0,1]^{J}$ is a solution to the equation $\bar{r}=g(\bar{r})$, where $g_{j}(\bar{r})=\min \left\{1, n_{j}(\bar{r}) \mu_{j} \bar{r}_{j}\right\} d_{j}\left(\bar{r}_{j}\right)$ for all $j=1, \ldots, J$. Hence, if $\bar{r}$ is a stationary equilibrium, then $h_{j}\left(\bar{r}_{j}\right)=\bar{r}_{j}$ for every $j \in J$ (where $\bar{r}_{\backslash j}$ is held fixed). But then, the statements in the theorem follow directly from Lemma 5 . II

From Theorem 1, we get simple conditions for the stationarity of the two extremal participation structures, zero participation and complete participation in all assets, respectively.

\section{Corollary 3.}

(i) $\bar{r}=(0, \ldots, 0)$ always is a stationary equilibrium.

(ii) For any non-redundant set of assets $K \subseteq J$, a phase $\bar{r} \in[0,1]^{J}$ with $\bar{r}_{j}=1$ for every $j \in K$ is a stationary equilibrium only if $n_{j}(\bar{r}) v_{j}(\bar{r}) \geqq 1$ for every $j \in K$. Moreover if $n_{j}(\bar{r}) v_{j}(\bar{r}) \geqq 1$ for every $j \in K$ and $\bar{r}_{j}=0$ for every $j \notin K$ then $\bar{r}$ is a stationary equilibrium.

(iii) Let $A$ be $\rho$-orthogonal. Then, for any non-redundant set of assets $K \subset J$, a phase $\bar{r} \in[0,1]^{J}$ with $\bar{r}_{j}=1$ for every $j \in K$ is a stationary equilibrium if and only if $\alpha_{j}^{J} \geqq 1$. Moreover, $\bar{r}_{j} \in(0,1)$ is part of a stationary equilibrium only if $\alpha_{j}^{J} m_{j}<1$, and $\alpha_{j}^{J} \bar{r}_{j}<1$ in which case $\bar{r}_{j}$ in unique.

Proof. Parts (i) and (ii) are obvious from Lemma 5 and Theorem 1. To see (iii) observe that if $A$ is $\rho$-orthogonal, then $n_{j}(\bar{r})=1$ by construction and $\mu_{j}\left(\bar{r}_{\backslash j}, \alpha_{j}\right)=\alpha_{j}^{J}$ by the proof of Lemma 3. Hence $n_{j}(\bar{r}) \mu_{j}=\alpha_{j}^{J}$. \|I

In order to determine the stability properties of stationary equilibria, we now compute the derivative of the transition function. 
Lemma 7. For any stationary equilibrium $\bar{r} \in[0,1]^{J}$ with $K_{+}(\bar{r})$ non-redundant and for almost all endowments $\omega$ we get:

(i) If $\bar{r}_{j} \in\{0,1\}$ then $\partial_{r_{j}} g_{j}(\bar{r})=\left\{\begin{array}{ll}m_{j} n_{j}(\bar{r}) \mu_{j}\left(r_{\backslash,}, \alpha_{j}\right) & \text { if } \bar{r}_{j}=0 \\ 1-m_{j}-w_{j} & \text { if } \bar{r}_{j}=1\end{array}\right.$.

(ii) If $\bar{r}_{j} \in\{0,1\}$ and $k \neq j$ then $\partial_{r_{k}} g_{j}(\bar{r})=\mathbf{0}$.

(iii) If $\bar{r}_{j} \in(0,1)$ then $\partial_{r_{j}} g_{j}(\bar{r})>1$.

Proof. Recall that $g_{j}(\bar{r})=d_{j}(\bar{r}) a_{j}(\bar{r})$, where $d_{j}\left(\bar{r}_{j}\right)=\bar{r}_{j}+m_{j}\left(1-\bar{r}_{j}\right)+w_{j} \bar{r}_{j}\left(1-\bar{r}_{j}\right)$ and $a_{j}(\bar{r})=\min \left\{1, n_{j}(\bar{r}) v_{j}(\bar{r})\right\}$ for every $j \in J$.

Compute

$$
\partial_{r_{k}} g_{j}(\bar{r})=\partial_{r_{k}} a_{j}(\bar{r}) d_{j}\left(\bar{r}_{j}\right)+a_{j}(\bar{r}) \partial_{r_{k}} d_{j}\left(\bar{r}_{j}\right)
$$

where

$$
\partial_{r_{k}} a_{j}(\bar{r})=\partial_{r_{k}} n_{j}(\bar{r}) v_{j}(\bar{r})+n_{j}(\bar{r}) \partial_{r_{k}} v_{j}(\bar{r}) \quad \text { if } n_{j}(\bar{r}) v_{j}(\bar{r})<1
$$

and

$$
\partial_{r_{k}} a_{j}(\bar{r})=0 \quad \text { if } n_{j}(\bar{r}) v_{j}(\bar{r})>1, \quad k, j \in J .
$$

(i) Suppose $k=j$ and $\bar{r}_{j}=0$. Then $a_{j}(\bar{r})=0$ since $v_{j}(\bar{r})=0$. Moreover, for the same reason $\partial_{r_{j}} a_{j}(\vec{r})=n_{j}(\vec{r}) \partial_{r_{j}} v_{j}(\bar{r})$. By Lemma $4, \partial_{r_{j}} v_{j}(\vec{r})=\mu_{j}\left(r_{\backslash j}, \alpha_{j}\right)$. Hence, $\partial_{r_{j}} g_{j}(\vec{r})=m_{j} n_{j}(\bar{r}) \mu_{j}\left(r_{\backslash j}, \alpha_{j}\right)$.

On the other hand, suppose $k=j$ and $\bar{r}_{j}=1$. Then $a_{j}(\vec{r})=1$ and $n_{j}(\bar{r}) v_{j}(\bar{r})=n_{j}(\vec{r}) \mu_{j}>1$ by Theorem 1(i), so that $\partial_{r_{j}} a_{j}(\bar{r})=0$. Hence, $\partial_{r_{j}} g_{j}(\bar{r})=1-m_{j}-w_{j}$.

(ii) Suppose $k \neq j$. Then, $\partial_{r_{k}} d_{j}\left(\bar{r}_{j}\right)=0$, since $d_{j}$ only depends on $r_{j}$. If $n_{j}(\bar{r}) v_{j}(\bar{r})>1$, then $\partial_{r_{k}} a_{j}(\bar{r})=0$. If $n_{j}(\bar{r}) v_{j}(\bar{r})<1$, then $a_{j}(\bar{r})<1$ and since $\bar{r}$ is a stationary equilibrium with $\bar{r}_{j} \in\{0,1\}$, it must follow that $\bar{r}_{j}=0$ by Theorem 1(i). But then, $v_{j}(\bar{r})=$ 0 and, by Lemma $4, \partial_{r_{k}} v_{j}(\vec{r})=0$, hence $\partial_{r_{k}} g_{j}(\bar{r})=0$.

(iii) Suppose $\bar{r}_{j} \in(0,1)$ is part of the stationary equilibrium. Observe that $\partial_{r,} g_{j}(\bar{r})=h_{j}^{\prime}\left(\bar{r}_{j}\right)$ where $\bar{r}_{V_{j}}=\left(\bar{r}_{1}, \ldots, \bar{r}_{j-1}, \bar{r}_{j+1}, \ldots, \bar{r}_{J}\right)$ is held fixed. Then Theorem 1 (ii) and Lemma 5 (iv) imply that $\partial_{r_{j}} g_{j}(\vec{r})>1$. \|

The stability properties of stationary equilibria are derived from Lemma 7 by application of some well known results of the Theory of Dynamical Systems. A stationary equilibrium is asymptotically stable if all the eigenvalues of the Jacobian of the transition function evaluated at the stationary point have absolute value less than 1 (see, for example, Devaney (1986)). Moreover, a stationary uniform participation phase $\bar{r}$ which is asymptotically stable with respect to $K_{t}(\vec{r})$ is evolutionarily stable with respect to the mutation of a new asset $j \notin K_{+}(\vec{r})$ if the diagonal entry corresponding to this mutation has absolute value less than 1 . This condition for evolutionary stability follows from Lemma 7. Consider a stationary point with $\bar{r}_{k}=0$. Since by Lemma $7, \partial_{r_{k}} g_{j}(\bar{r})=0$ for all $j$ with $\bar{r}_{j} \in\{0,1\}$, the Jacobian matrix $\partial_{r} g$ is a diagonal matrix at any uniform participation phase, and therefore, in a small neighbourhood around $\bar{r}$, the derivative of $g$ in the direction of $k$ is given by $\partial_{r j} g_{j}(\vec{r})$. A similar argument applies for the stability with respect to small reductions of participation levels below 1, i.e. for an asset's property to remain perfect. 
Therefore, we get the following stability properties:

Theorem 2. At any stationary equilibrium $\bar{r} \in\{0,1\}^{J}$ with $K_{+}(\bar{r})$ non-redundant, and for almost all endowments $\omega$, we get:

(i) If $\bar{r}$ is asymptotically stable with respect to $K_{+}(\bar{r})$ then $\bar{r}$ is evolutionarily stable with respect to the innovation of a non-redundant asset $j \notin K_{+}(\vec{r})$ if $m_{j} n_{j}(\vec{r}) \mu_{j}<1$.

(ii) If $\bar{r}$ is asymptotically stable with respect to $K_{+}(\bar{r})$ then $\bar{r}$ is not evolutionarily stable with respect to the innovation of a non-redundant asset $j \notin K_{+}(\bar{r})$ if $m_{j} n_{j}(\bar{r}) \mu_{j}>1$.

(iii) If $\bar{r}$ with $\bar{r}_{j}=1$ is asymptotically stable with respect to $K_{+}(\bar{r}) \backslash\{j\}$ then the participation structure remains perfect with respect to asset $j$.

Moreover any stationary equilibrium $\bar{r}$ with $\bar{r}_{j} \in(0,1)$ for some $j \in J$ is not asymptotically stable.

Proof. First assume that $\bar{r} \in\{0,1\}^{J}$. Then, by part (ii) of Lemma 7 , the directional derivative of $g$ in the $k$-th direction is given by the $k$-th diagonal element of $\partial_{r} g(\bar{r})$. Hence, part (i) of Lemma 7 together with Lemma 5 gives the stability conditions (i), (ii), and (iii).

To show the last statement assume that $\bar{r}$ is stationary and that there is some $k \in J$ such that $\bar{r}_{k} \in(0,1)$. Recall from elementary linear algebra that the trace of a matrix is equal to the sum of its eigenvalues. Part (ii) of Lemma 7 implies for every $j \in J$ with $j \in$ $\{0,1\}$ that the $j$-th unit vector is a left eigenvector of $\partial_{r} g(\vec{r})$ with corresponding eigenvalue $\partial_{r_{j}} g_{j}(\bar{r})$. Since furthermore $\partial_{r_{k}} g_{k}(\bar{r})>1$ for $k$ with $\bar{r}_{k} \in(0,1)$, at least one eigenvalue of $\partial_{r} g(\bar{r})$ must be greater than one. Hence $\bar{r}$ is not asymptotically stable. \|

Moreover, as the next theorem shows, a stationary equilibrium with full participation in a complete set of markets is not only evolutionarily stable but also asymptotically stable. If the economy happens to settle in a complete set of markets it cannot be unsettled by small perturbations to the participation rates.

Theorem 3. Any stationary equilibrium $\bar{r} \in\{0,1\}^{J}$ with $K_{+}(\bar{r})$ forming a non-redundant and complete set of markets is asymptotically stable.

Proof. To demonstrate asymptotic stability first note that by Lemma $7, \partial_{r} g(\bar{r})$ is a diagonal matrix because by assumption of Theorem 3 for all $j \in J$ either $\bar{r}_{j}=0$ or $\bar{r}_{j}=1$. Hence locally the transition function $g$ decomposes into $J$ independent functions. Therefore $\bar{r}$ is asymptotically stable if every $j \in K_{+}(\bar{r})$ remains perfect and if $\bar{r}$ is evolutionarily stable with respect to any $j \notin K_{+}(\bar{r})$. The former is indeed the case because for $\bar{r}=1$ the $j-$ th diagonal element is $1-m_{j}-w_{j}$ which is smaller than one. The latter requires to extend $g$ on a neighbourhood of $\bar{r}$ to redundant assets. Such an extension which keeps the utility maximization property of asset demand and which does not destroy the differentiability of $g$ is always possible, as the following simple example demonstrates.

Let $\tilde{J}$ be such that $\tilde{J} \cap K_{+}(\tilde{r})=\varnothing$ be a set of possible financial innovations, each of which is redundant to $K_{+}(\tilde{r})$, i.e.

$$
A_{j}=\sum_{k \in K_{+}(\bar{r})} A_{k} \bar{\theta}_{k}^{j}, \quad j \in \tilde{J} .
$$

Now with reference to Definition 3 consider the recursion

$$
\begin{aligned}
r_{k}(t+1)=\bar{r}_{k}-\sum_{j \in J} \bar{\theta}_{k}^{j}, r_{j}(t), & \text { with } r_{k}(0)=\bar{r}_{k}, t=0,1,2, \ldots, k \in K_{+}(\vec{r}), \\
r_{j}(t+1)=1 /(t+1) r_{j}(0), & \text { with } r_{j}(0)=\varepsilon_{j}, t=0,1,2, \ldots, j \in \tilde{J} .
\end{aligned}
$$


Note that $\lim _{t \rightarrow \infty}=\bar{r}$. Finally note that for any such extension $\bar{r}$ is evolutionarily stable because the innovation coefficient, $n_{j}(\bar{r})$, is zero for all $j \notin K_{+}(\vec{r})$. Hence, $\bar{r}$ is indeed asymptotically stable. II

Finally, in the case of $\rho$-orthogonal assets the criterion for evolutionary stability is independent of the particular equilibrium.

Corollary 4. Let the assets be $\rho$-orthogonal. Then a stationary equilibrium $\bar{r} \in[0,1]^{J}$ with $K_{+}(\vec{r})$ non-redundant is evolutionarily stable with respect to the innovation of asset $j \notin K_{+}(\bar{r})$ if $\alpha_{j}^{J} m_{j}<1$. If $\alpha_{j}^{J} m_{j}>1$, then $\bar{r}$ is not evolutionarily stable with respect to the innovation of asset $j$. Moreover, $\bar{r}$ is asymptotically stable if and only it it is evolutionarily stable.

Proof. By construction $n_{j}(\vec{r})=1$ for all $j \in J$ if assets are $\rho$-orthogonal. Moreover, by Lemma 3, the equilibrium volume of trade of any asset is independent of the other assets' market participation. Hence, $\partial_{r} g(\bar{r})$ is a diagonal matrix, and its stability properties then are determined by its diagonal entries. \|

The simple stability properties of stationary equilibria derived in Theorems 2 and Theorem 3 and in Corollary 4 rely on two particular features of the transition process:

Firstly, whenever a stationary equilibrium $\bar{r}$ corresponds to a standard GEI-economy, i.e. whenever $\bar{r} \in\{0,1\}$, then $\bar{r}$ can locally be characterized by the one-dimensional dynamics described by $h_{j}$ for every $j \in J$. In particular, the stability of $\bar{r}$ only depends on the slope of $h_{j}$ at $\bar{r}_{j}=0$ and $\bar{r}_{j}=1$. The derivative $h_{j}^{\prime}(0)$ depends on the marketing coefficient $m_{j}$, the coefficient of asset $j$ 's trading volume $\mu_{j}\left(\bar{r}_{\backslash}, \alpha_{j}\right)$, and the degree of asset $j$ 's "innovativeness" $n_{j}\left(\bar{r}_{\backslash}, 0\right)$. If, for example, there is no marketing effort, ${ }^{33}$ i.e. $m_{j}$ small, or if asset $j$ is almost redundant $\left(n_{j}\right.$ small $)$ or if it does not generate a sufficiently high trading volume ( $\mu_{j}$ is small), then evolutionary stability with respect to asset $j$ follows, i.e. asset $j$ is a "failure". On the other hand, the derivative $h_{j}^{\prime}(1)$ will always be smaller than 1 since if $\bar{r}_{j}=1$ is a part of a stationary equilibrium then $h_{j}$ necessarily is identical to $d_{j}$ in a neighbourhood of 1 (for "regular economies", i.e. for almost all endowments), where $d_{j}^{\prime}(1)=$ $1-m_{j}-w_{j}<1$. But the fact that the cross-derivatives do not matter in this case (Lemma 7(ii)) implies that asset $j$ must be remaining perfect. Hence, any small reduction of market participation rates of assets in which there is full participation in the stationary equilibrium will lead back to full participation.

Secondly, the economy cannot get stuck in a situation of mixed market participation, where potential spill-over effects might become relevant (i.e. where the cross-derivatives can be non-zero): In such a situation, eventually, and after any perturbation, every asset will either be adopted by the entire economy or it will disappear. As can be seen from the proof of Lemma 7(iii), this instability is implied by the important fact from linear algebra that the trace of a matrix is equal to the sum of its eigenvalues. Based or this observation, the lack of stability of mixed participation equilibria can be deduced from the shape of the one-dimensional functions $h_{j}$-in spite of the fact that the transition function is multidimensional in any neighbourhood around the mixed participation equilibrium. As a consequence, the "standard" GEI-economies are the only stable stationary outcomes of the dynamical process modelled in this paper, and they are characterized by simple, locally one-dimensional, dynamics.

33. It is commonly found in the empirical literature that marketing is among the key factors that distinguishes top performing products in financial services (see Cooper, Easingwood, Edgett, Kleinschmidt and Storey (1994)). 


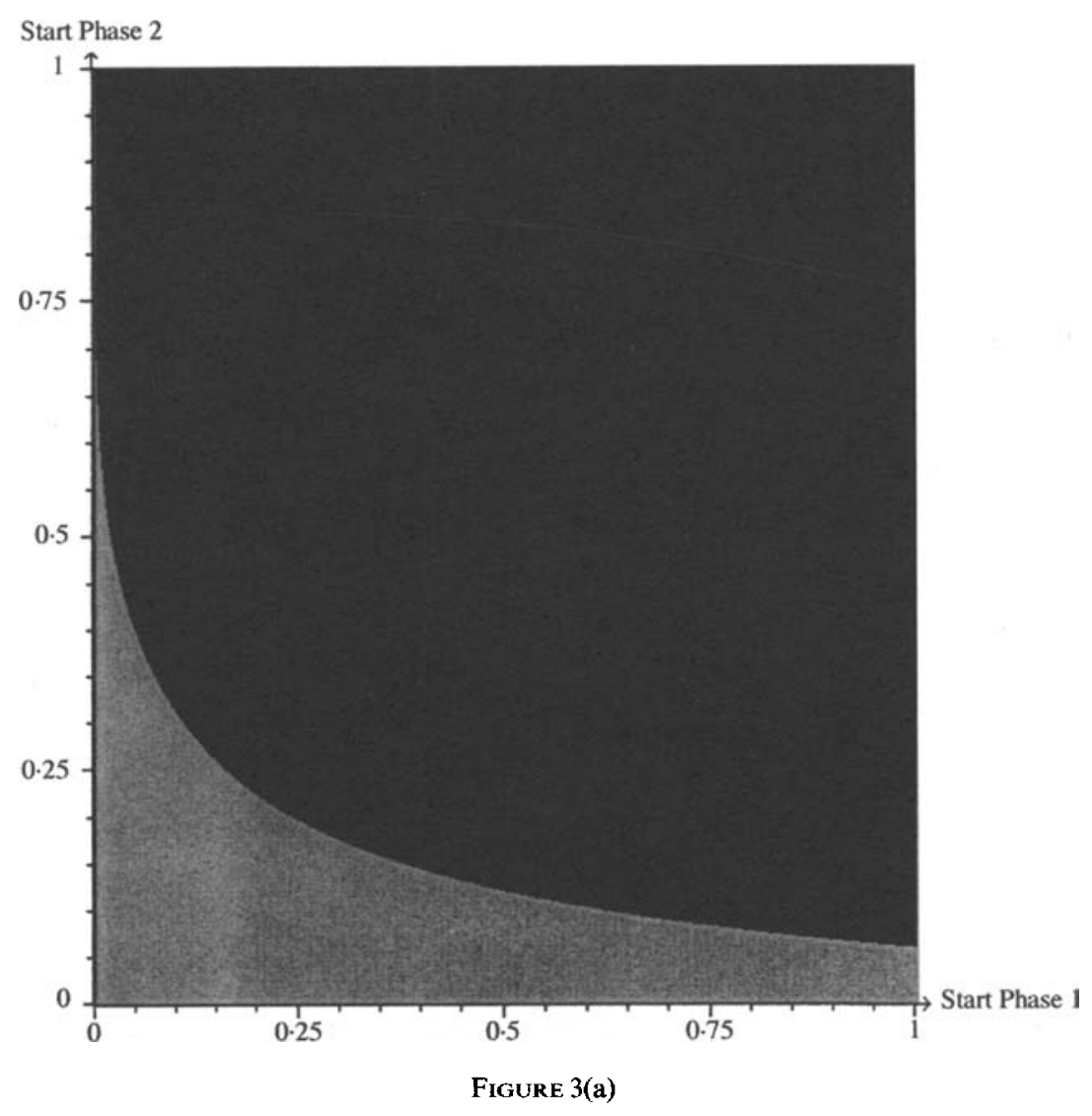

Obviously, the simplicity of the results achieved depends on the restrictiveness of the assumptions made. If any of the assumptions were relaxed then an explicit characterization of the transition dynamics would soon become inherently difficult. In this case, one would have to turn towards computer simulations and numerical analysis. We have used such methods already in the situation considered in this paper, in order to further illustrate our model by means of some interesting examples. We will present these examples in the following subsection. ${ }^{34}$ Besides illustrating the general predictions of our model and indicating a potential approach to more difficult specifications of the stage economies and the transition process, the simulation programme also allows to derive new results. Whereas, for example, our results in the general model characterize the local stability of stationary equilibria, the simulation programme allows to visualize the basins of attraction of stationary equilibria which often turn out to be much larger than some small $\varepsilon$-neighbourhood. Moreover, the simulation programme allows to show the trajectories of the dynamical process, starting at arbitrary initial values. These trajectories display some interesting non-monotonic dynamics which could not have been concluded from our general results.

34. The examples are obtained from a simulation programme written by our research assistant Jan Pilgrim. The programme is based on the simulation programme MACRODYN created in the research group of Volker Böhm and described in Böhm and Schenk-Hoppé (1998). We are grateful for this support. 


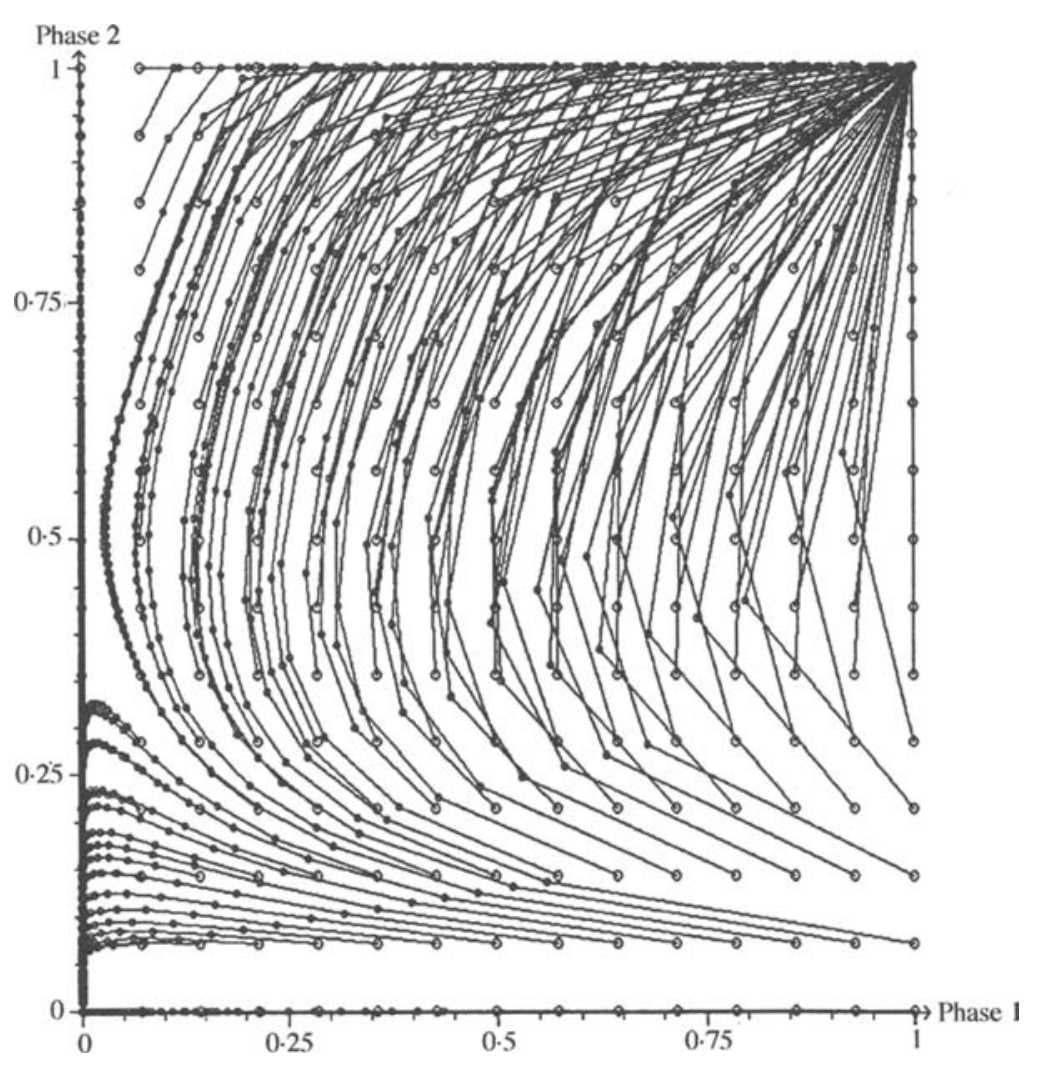

Figure 3(b)

\subsection{Numerical examples}

The examples presented in this section illustrate the case of "nuts and bolts", the possibility of stationary mixed participation equilibria, the phenomenon of "path-dependence", and, finally, the possibility that an initially successful asset is later driven out of the market by some other, even more successful asset.

The first example illustrates the famous case of "nuts and bolts". Hart (1979) has suggested an example, where a coordination failure between innovating intermediaries may lead to an inefficient outcome of the innovation process. In a different framework, Hart's example is repeated by Heller (1993) and by Che and Rajan (1994). The idea behind this example can be described as follows. Suppose there are two market makers who can open a market for a certain asset at some fixed cost. Market makers recover these fixed costs through transaction costs they charge for each unit of the asset traded. Suppose furthermore that the two assets possibly traded on the two markets are complements with respect to trading volume. In that case, every asset generates a low trading volume if introduced while the market for the other asset remains closed; if both markets are opened simultaneously, however, then trading volume in both assets will be high. Hart (1979), Heller (1993), and Che and Rajan (1994) now point out that if fixed costs for the opening of a market are sufficiently high, both market makers may prefer to keep their market closed because each market maker expects the other one to do so. Due to this coordination 


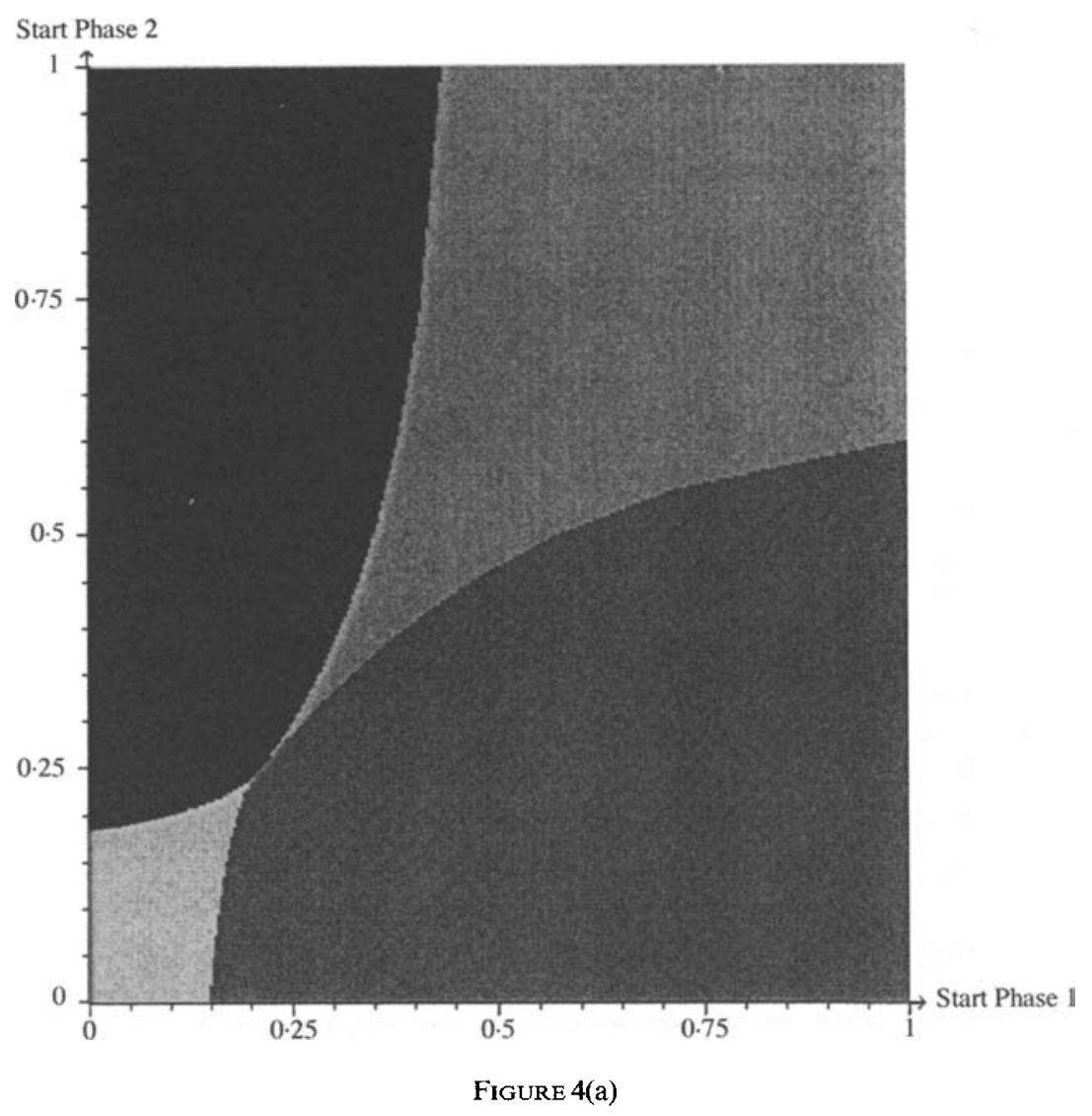

failure the economy might therefore get stuck in the inefficient no trade-equilibrium without any available assets. For obvious reasons, this example is usually referred to as the "nuts and bolts" example.

In our example two assets can be complementary in such a way that the innovation of only one of them is not successful. In our model, however, both assets only get started if they are introduced simultaneously and with a level of participation which exceeds a certain threshold in the open interval $(0,1){ }^{35}$ Note that this interesting aspect of financial innovation cannot be analysed in the traditional "nuts and bolts" examples since innovating in these models means to increase participation rates abruptly from zero to one.

The characteristics of the economy in our version of the "nuts-and-bolts" example are given by:

Example 1. $I=2, \quad J=2, \quad S=2, \quad \rho_{1}=0.5, \quad \rho_{2}=0.5, \quad \gamma^{1}=0.6, \quad \gamma^{2}=0 \cdot 4, \quad \omega^{1}=$ $(1, \sqrt{2}, 0 \cdot 125)^{T}, \omega^{2}=(1,0 \cdot 125, \sqrt{2})^{T}, A_{1}=(1,1)^{T}, A_{2}=(0, \sqrt{2})^{T}, m_{1}=1, m_{2}=1, w_{1}=0$, $w_{2}=0$.

Figure 3(a) shows the basins of attraction for Example 1, while Figure 3(b) shows trajectories of the dynamical process implied by Example 1. ${ }^{36}$

35. In Example 1, introducing both assets with participation rates $r_{1}(0)=r_{2}(0)=0.25$ suffices to get both markets started, whilst choosing $r_{1}(0)=r_{2}(0)=0.2$ will cause both assets to eventually die out.

36. In these and the following figures, the horizontal (vertical) axis displays the participation rate of the first asset, i.e. $r_{1}$ (the participation rate of the second asset, i.e. $r_{2}$ ). Different basins of attraction are shaded differently. Finally, trajectories read as moving from dot to dot. 


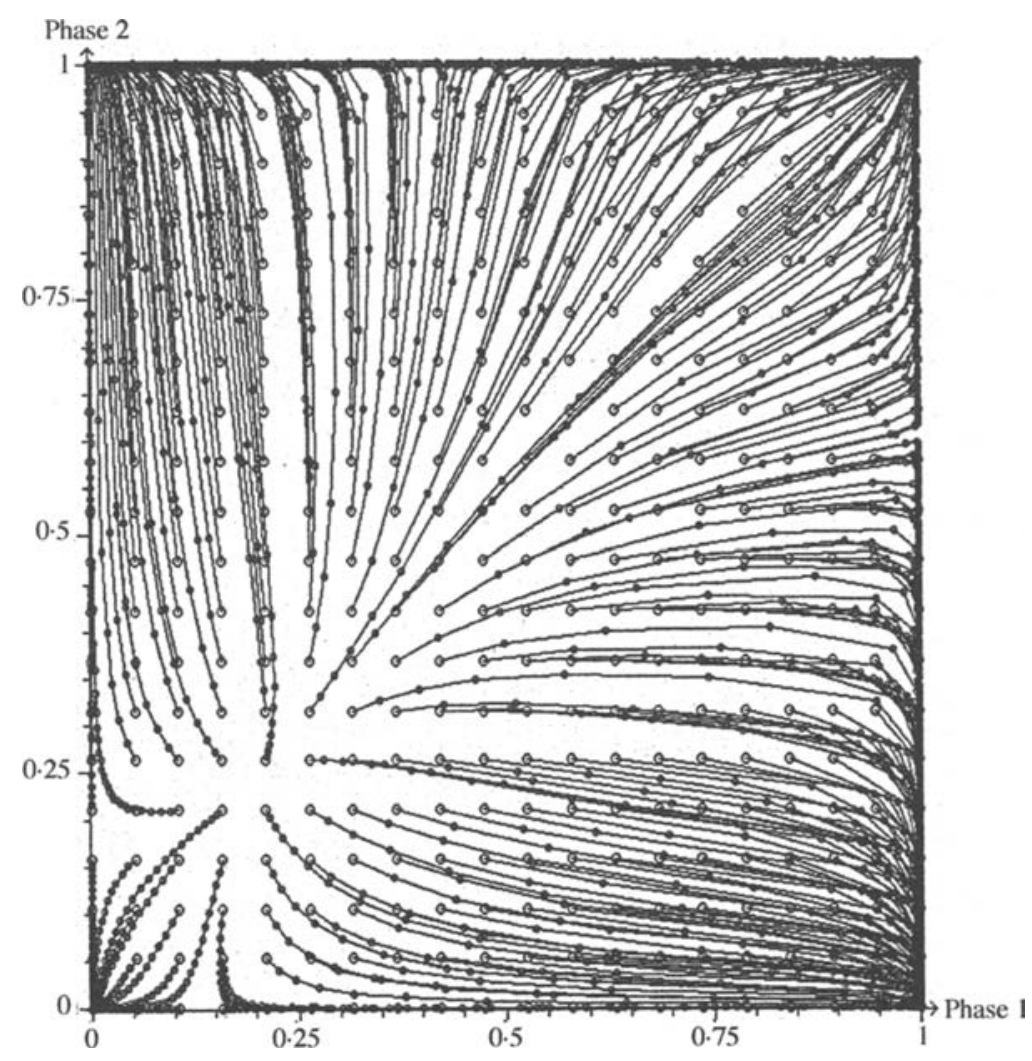

FIGURE 4(b)

The next example shows the possibility of a mixed participation equilibrium. The characteristics of the economy in Example 2 are:

Example 2. $I=2, J=2, S=3, \rho_{1}=1 / 3, \rho_{2}=1 / 3, \rho_{3}=1 / 3, \gamma^{1}=0 \cdot 4, \gamma^{2}=0 \cdot 3, \omega^{1}=$ $(1,2,0,0)^{T}, \quad \omega^{2}=(1,0,0,2)^{T}, \quad A_{1}=(\sqrt{1 \cdot 5},-\sqrt{1 \cdot 5}, 0)^{T}, A_{2}=(0, \sqrt{0 \cdot 6}, \sqrt{2 \cdot 4})^{T}, m_{1}=0 \cdot 5$, $m_{2}=0.5, w_{1}=0.4, w_{2}=0.4$.

In Figure 4(a) there is a mixed equilibrium at the point where the basins of attraction of the four uniform participation equilibria "kiss" each other. Figure 4(b) nicely illustrates the instability of the mixed participation equilibrium. Note that there are mixed participation equilibria also at $r=\left(\bar{r}_{1}, 0\right)$ and $r=\left(0, \bar{r}_{2}\right)$. These stationary equilibria are not asymptotically stable since slight variations in own participation cause the participation phase to divert from them (as predicted by Theorem 2), moving either towards full or zero participation. In this case, these mixed participation equilibria on the boundary also are evolutionarily unstable. ${ }^{37}$

Example 3 illustrates the phenomenon of "path dependence" or "lock-in" which often occurs in evolutionary models with network externalities (see, e.g. Arthur (1989) or

37. Note that assets $A_{1}$ and $A_{2}$ are not $\rho$-orthogonal so that the last statement of Corollary 4 is not contradicted. 


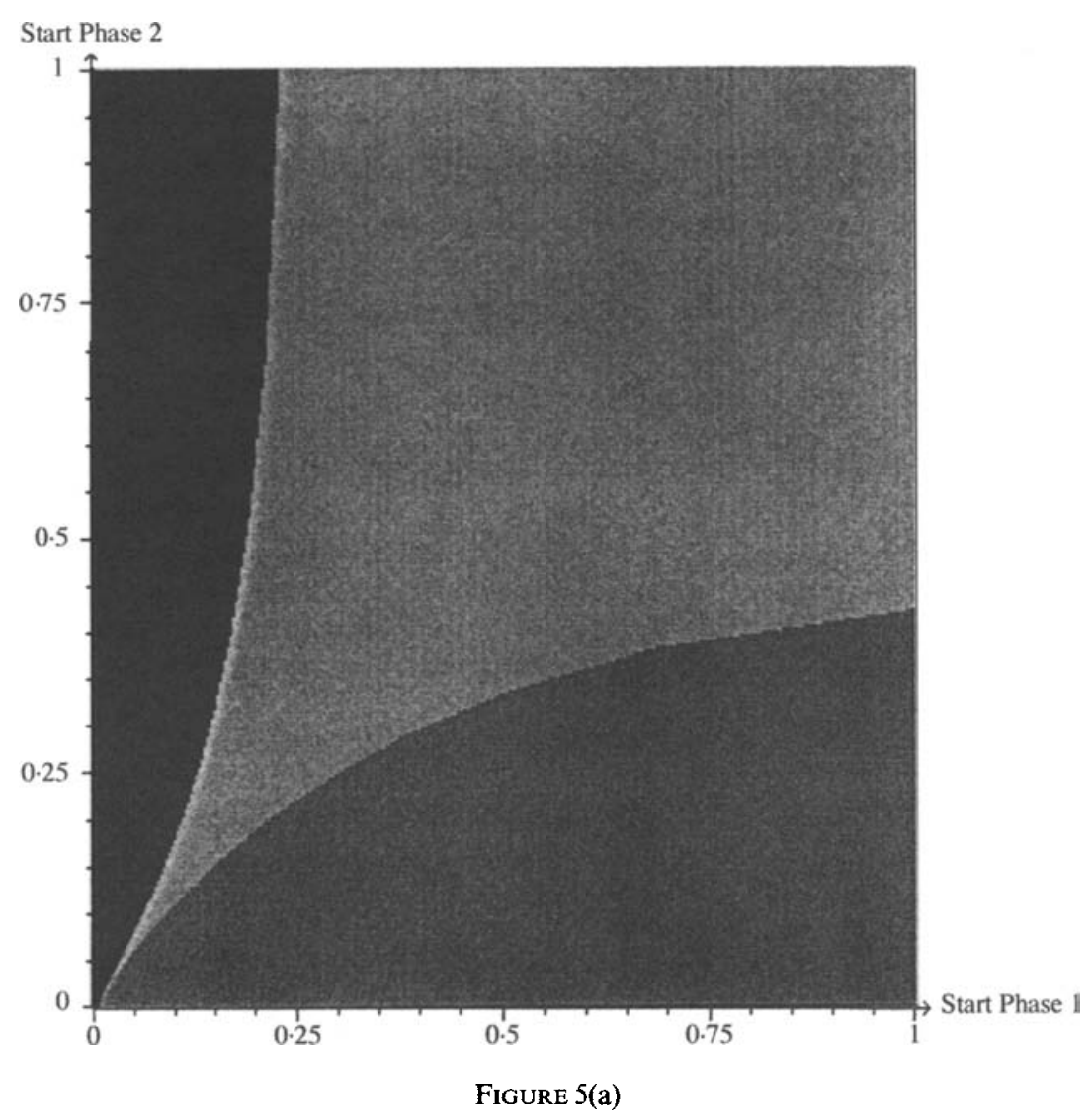

David (1993)). If the innovation path were started with the introduction of asset 1 (asset 2 ), it would end at the stationary point in which only asset 1 (asset 2) is traded.

The characteristics of Example 3 are:

Example 3. $I=2, J=2, S=3, \rho_{1}=1 / 3, \rho_{2}=1 / 3, \rho_{3}=1 / 3, \gamma^{1}=0 \cdot 4, \gamma^{2}=0 \cdot 3, \omega^{1}=$ $(1,2,0,0)^{T}, \quad \omega^{2}=(1,0,0,2)^{T}, \quad A_{1}=(\sqrt{1 \cdot 5},-\sqrt{1 \cdot 5}, 0)^{T}, \quad A_{2}=(0, \sqrt{0 \cdot 6}, \sqrt{2 \cdot 4})^{T}, \quad m_{1}=0 \cdot 7$, $m_{2}=0 \cdot 7, w_{1}=0 \cdot 3, w_{2}=0 \cdot 3$.

Figure 5(a) shows the corresponding basins of attraction while Figure 5(b) displays some trajectories.

The last example illustrates how an asset can be driven out of the market even though every asset "remains perfect" (see Theorem 2). This can happen if a stationary equilibrium is not evolutionarily stable with respect to the innovation of some new asset, and if, in the presence of the new asset, some of the former assets do no longer generate sufficient trading volume to remain a part of a stationary equilibrium.

Example 4. $I=2, J=2, S=2, \rho_{1}=0.5, \rho_{2}=0 \cdot 5, \gamma^{1}=0 \cdot 25, \gamma^{2}=0.25, \omega^{1}=(1,3,0)^{T}$, $\omega^{2}=(1,0,3)^{T}, A_{1}=(\sqrt{2}, 0)^{T}, A_{2}=(1,-1)^{T}, m_{1}=0.5, m_{2}=0.5, w_{1}=0.5, w_{2}=0.5$.

The basin of attraction of the point $(0,1)$ contains the whole square $(0,1)^{2}$. Figure 6 illustrates the dynamics of "being driven out" by some trajectories of the dynamical process. 


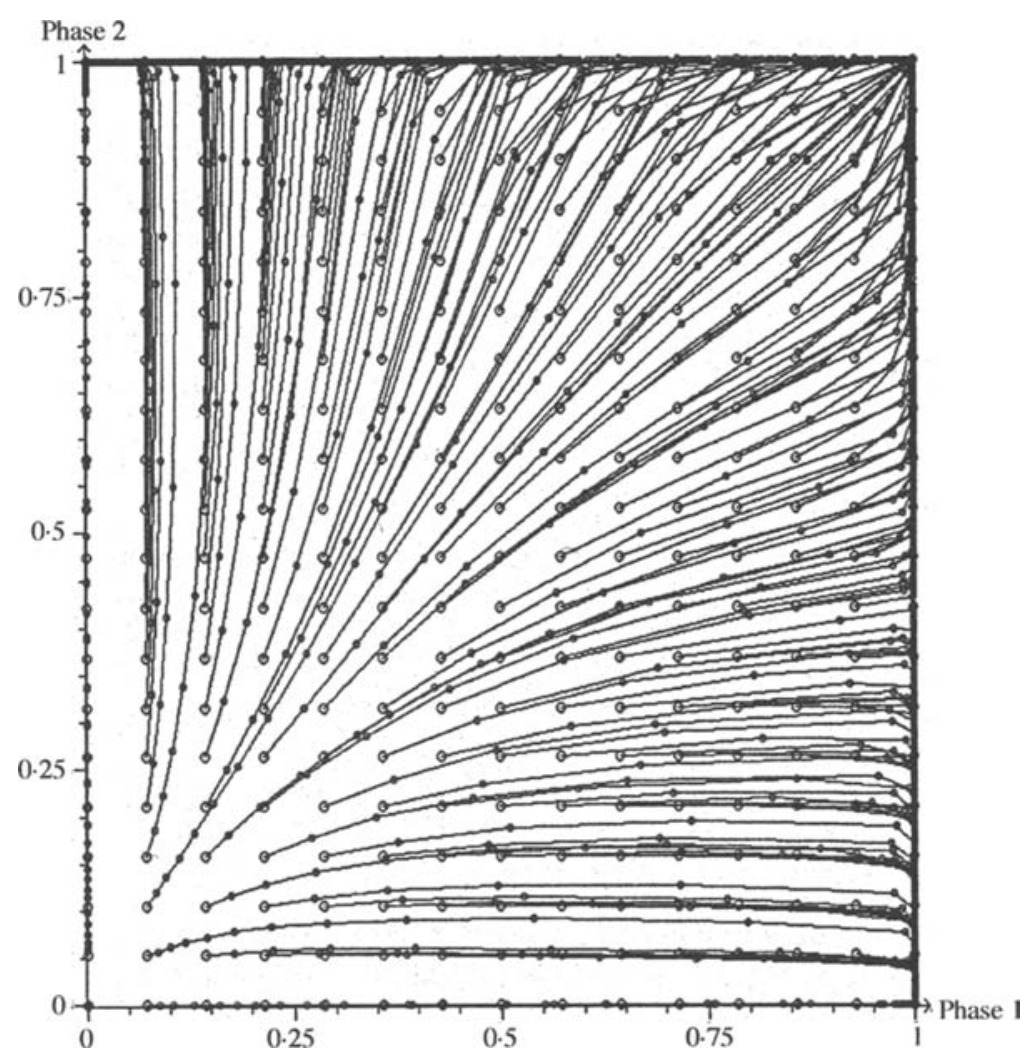

FIGURE 5(b)

\section{CONCLUSION}

This paper has presented a novel evolutionary approach to the analysis of innovation in financial markets. We have superimposed an evolutionary process on to a GEI-model with restricted participation and have shown that the stationarity and stability notions known from other branches of evolutionary economics nicely carry over to our setting. Our results characterize stationary equilibria and give a complete characterization of their evolutionary and asymptotic stability properties. Being based on bounded rationality, these results are well in line with the empirical literature on the reasons for the success and the failure of financial innovations.

This paper could only give an introductory treatment of the evolutionary approach to financial innovation. Further research will have to be conducted along two main lines.

On the one hand, the simple structure of the model presented could be extended in various directions. Firstly, the approach could be applied to examples other than the CAPM. Secondly, further effort could extend the model towards cases not using the simplifying independence assumptions. Finally, it might be considered whether consecutive time periods could be linked by more than just the asset dynamics, for example by feedback effects of asset trades on the agents' endowments, by longer-lived assets, and by more general asset payoffs.

On the other hand, it should be attempted to endogenize the transition function between the stage economies. As a first step in this direction, Güth and Ludwig (2000) 


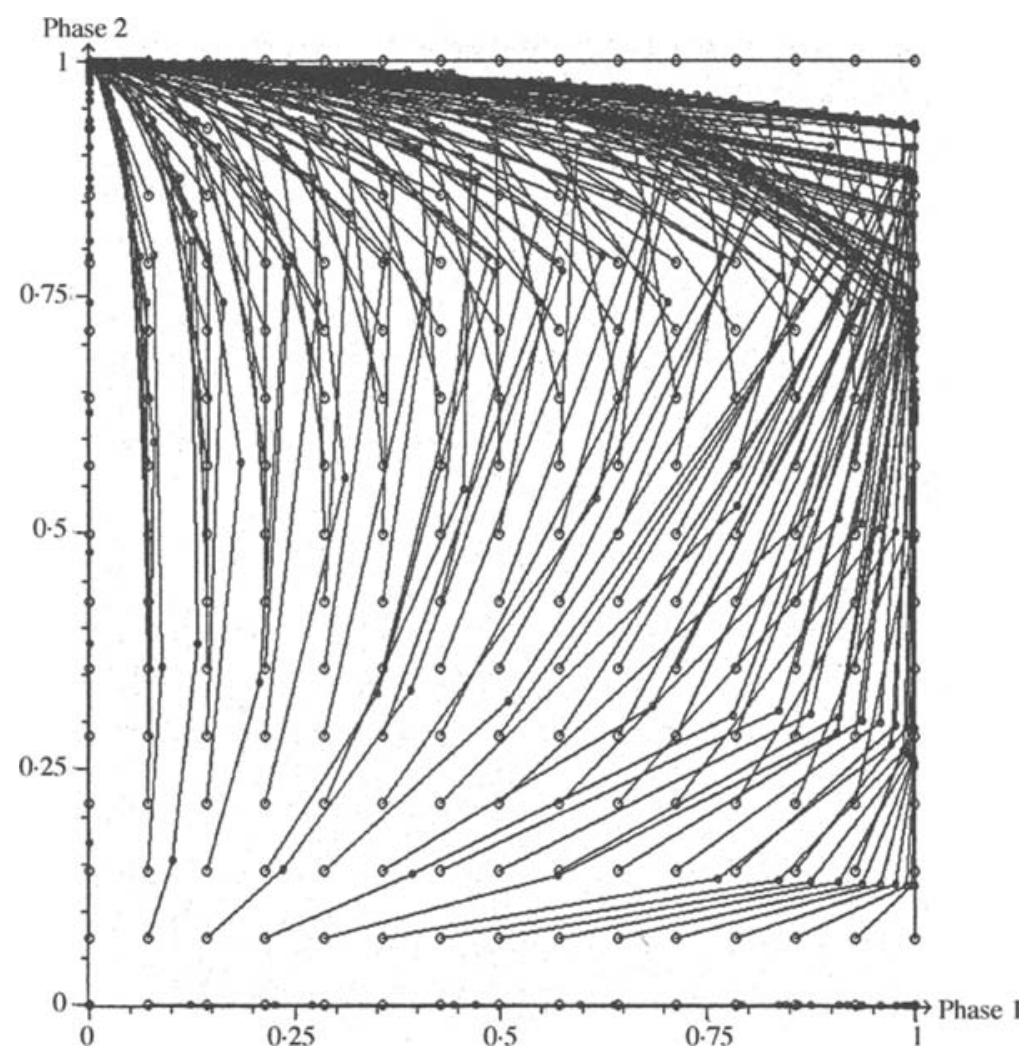

Figure 6

modify our paper by modelling a process of social learning in a sequence of CAPMeconomies with (endogenously) restricted participation. Their approach allows to explicitly derive the transition of market participation rates from one period to the next; in their model, this transition is based on expected utility comparisons in the sense of imitation and experimentation. It turns out that this transition process will naturally converge to complete markets-at least, as assumed in our paper, if there are no a priori upper bounds on the number of assets an agent can participate in.

Acknowledgements. We wish to thank I. Evstigneev, A. Gerber, A. Kirman, J.-J. Herings, W. Hildenbrand, K. Schenk-Hoppé, F. Vega-Redondo, B. Zame and seminar participants in Bergen, Berlin, Bonn, Bielefeld, Cambridge, CORE, Lausanne, Mannheim, Tilburg, Berkeley, USC, Stanford and the participants of the Geneva-Venice workshop on Incomplete Markets in Venice as well as of ESEM '96 in Istanbul, Antalya '97, SITE ' 97 for helpful comments. Financial support by the Swiss Stock Exchange as well as by Deutsche Forschungsgemeinschaft through Sonderforschungsbereich 303 at the University of Bonn and through the research project "Evolution in Financial Markets" at the University of Bielefeld is gratefully acknowledged. Last but not least we are grateful for many very useful suggestions by the referees of the Review. All remaining errors are ours.

\section{REFERENCES}

ADMATI, A. R, and PFLEIDERER, P. (1988), “A Theory of Intraday Patterns: Volume and Price Variability", Review of Financial Studies, 1, 3-40.

ALLEN, F. and GALE, D. (1994) Financial Innovation and Risk Sharing (Cambridge: MIT Press). 
ARTHUR, W. B. (1989), “Competing Technologies, Increasing Returns, and Lock-in by Historical Events", Economic Journal, 99, 116-131.

BALASKO, Y., CASS, D. and SICONOLFI, P. (1990), "The Structure of Financial Equilibrium with Exogenous Yields: The Case of Restricted Participation", Journal of Mathematical Economics, 19, 195-216.

BIKHCHANDANI, S., HIRSHLEIFER, D. and WELCH, I. (1998), "Leaming from the Behaviour of Others: Conformity, Fads, and Informational Cascades", Journal of Economic Perspectives, 12, 151-170.

BISIN, A. (1998), "General Equilibrium Economies with Imperfectly Competitive Financial Intermediaries" Journal of Economic Theory, 82, 19-45.

BLACK, D. (1986), "Success and Failure of Futures Contracts: Theory and Empirical Evidence" (Monograph Series in Finance and Economics, New York University).

BÖHM, V. and SCHENK-HOPPÉ, K. R. (1998), “MACRODYN-A User's Guide” (Discussion Paper No. 400, Department of Economics, University of Bielefeld).

CHE, J. and RAJAN, U. (1994), "Endogenous Financial Market Formation in a General Equilibrium Model" (Working Paper, Department of Economics, Stanford University).

COOPER, R. G., EASINGWOOD, C. J., EDGETT, S., KLEINSCHMIDT, E. J. and STOREY, C. (1994) "What Distinguishes the Top Performing New Products in Financial Services?" Journal of Product Innovation and Management, 11, 281-299.

CORCHÓN and MAS-COLELL, A. (1996), "A Note on Stability of Best Reply and Gradient Systems with Applications to Imperfectly Competitive Models", Economics Letters, 51, 59-65.

CUNY, C. J. (1993), "The Role of Liquidity in Futures Market Innovations", Review of Financial Studies, 6, $57-78$.

DAVID, P. A. (1993), "Path-Dependence and Predictability in Dynamical Systems with Local Network Externalities: A Paradigm for Historical Economics", in D. Foray and C. Freeman (eds.), Technology and the Wealth of Nations (London: Printer) 208-231.

DEKEL, E., LIPMAN, B. L. and RUSTICHINI, A. (1998), "Standard State-Space Models Preclude Unawareness Econometrica, 66, 159-173.

DEVANEY, R. C. (1984) An Introduction to Chaotic Dynamical Systems (Menlo Park: Benjamin-Cunnings).

DUFFIE, D. (1988) Security Markets: Stochastic Models (San Diego: Academic Press).

DUFFIE, D. and JACKSON, M. O. (1989), "Optimal Security Design", Review of Financial Studies, 2, 275 296.

DUFFIE, D. and RAHI, R. (1995), “Financial Market Innovation and Security Design”, Journal of Economic Theory Symposium on Financial Innovation and Security Design, 65, 1-42.

GÜTH, S. and LUDWIG, S. (2000), "The Evolutionary Emergence and Vanishing of Assets in Incomplete Financial Markets" (Discussion Paper, University of Bielefeld).

HARA, C. (1995), "On the Commission-Revenue Maximization in a General Equilibrium Model of Asset Creation", Journal of Economic Theory, 65, 258-298.

HART, O. (1979), "Monopolistic Competition in a Large Economy with Differentiated Commodities", Review of Economic Studies, 46, 1-30.

HELLER, W. (1993), "Equilibrium Market Formation Causes Missing Markets" (Working Paper, Department of Economics, University of California at San Diego).

HENS, T., SCHMEDDERS, K. and VOSS, B. (1999), "On Multiplicity of Competitive Equilibria when Markets are Incomplete", in Herings et al. (eds.), The Theory of Markets (Amsterdam: North-Holland).

HILDENBRAND, W. and KIRMAN, P. (1988) Equilibrium Analysis (Amsterdam: North-Holland).

HOPENHAYN, H. A. and WERNER, I. M. (1996), "Information, Liquidity and Asset Trading in a Random Matching Game", Journal of Economic Theory, 68, 349-379.

KYLE, A. S. (1985), "Continuous Auctions and Insider Trading", Econometrica, 53, 1315-1335.

KYLE, A. S. (1989), "Informed Speculation with Imperfect Competition", Review of Economic Studies, 56, $312-355$.

MAGILL, M. and QUINZII, M. (1996) Theory of Incomplete Markets (Cambridge: MIT Press).

MERTON, R. C. (1987), "A Simple Model of Capital Market Equilibrium with Incomplete Information", Journal of Finance, 42, 483-510.

MILLER, M. H. (1986) Financial Innovations and Market Volatility (Cambridge: Blackwell).

MILLER, M. H. (1992), "Financial Innovation: Achievements and Prospects", Journal of Applied Corporate Finance, 4, 8-10.

OH, G. (1994), "Some Results in the CAPM with Non-Traded Endowments" (Research Paper, Financial Markets Institute, Yale University).

PADOLSKI, T. (1987), "Financial Innovation and Money Supply", in Eatwell et al. (eds.), The New Palgrave on Money and Finance (London: Macmillan).

PAGANO, M. (1989a), "Trading Volume and Asset Liquidity", Quarterly Journal of Economics, 104, $255-274$.

PAGANO, M. (1989b), "Endogenous Stock Market Thinness and Stock Price Volatility", Review of Economic Studies, 56, 269-288.

PESENDORFER, W. (1995), "Financial Innovation in a General Equilibrium Model", Journal of Economic Theory, 65, 79-116.

PILGRIM, J. (1998), "Eine numerische Analyse evolutionärer Finanzmärkte” (Diploma-Thesis, University of Bielefeld). 
POLEMARCHAKIS, H. and SICONOLFI, P. (1997), "Generic Existence of Competitive Equilibria with Restricted Participation", Journal of Mathematical Economics, 28, 289-311.

ROGERS, E. M. (1995) Diffusion of Innovations (New York: The Free Press).

ROGERS, E. M. and SCHOEMAKER, F. F. (1971) Communication of Innovations: A Cross-Cultural Approach (New York: The Free Press).

SICONOLFI, P. (1986), "Equilibrium with Restricted Participation in Incomplete Markets (Mimeo, University of Pennsylvania)

SICONOLFI, P. (1989), "Equilibrium with Asymmetric Constraints on Portfolio Holdings and Incomplete Financial Markets", in M. Galeotti, L. Geronazzo and F. Gori (eds.), Nonlinear Dynamics in Economics and Social Sciences (Societá Pitagora, December 1989) 271-292.

SILBER, W. L. L. (1975) Financial Innovations (New York: The Free Press).

SIMON, H. A. (1955), "A Behavioral Model of Rational Choice", Quarterly Journal of Economics, 69, 99-118. SIWIK, T. (2000), "Theoretical Foundations of the CAPM" (Mimeo, University of Bielefeld).

THE ECONOMIST (1996), "Too Hot to Handle, A Survey of Corporate Risk Management" (Supplement to Vol. 338, No. 7952).

TUFANO, P. (1989), "Financial Innovation and First-Mover Advantage", Journal of Financial Economics, 25, $213-240$.

WILLIAMSON, S. D. (1996), "Liquidity and Market Participation", Joumal of Economic Dynamics and Control, 18, 629-670. 